\title{
How do Enzymes Utilize Reactive OH Radicals? Lessons from \\ Non-Heme HppE and Fenton Systems
}

\author{
Binju Wang* $*^{\dagger}$, Jiarui Lu ${ }^{\ddagger}$, Kshatresh Dutta Dubey ${ }^{\dagger}$, Geng Dong ${ }^{\ddagger}$, Wenzhen Lai* ${ }^{\ddagger}$, \\ Sason Shaik*广 \\ ${ }^{\dagger}$ Institute of Chemistry and The Lise Meitner-Minerva Center for Computational Quantum \\ Chemistry, The Hebrew University of Jerusalem, 91904, Jerusalem, Israel. \\ ${ }^{ \pm}$Department of Chemistry, Renmin University of China, Beijing, 100872, China
}

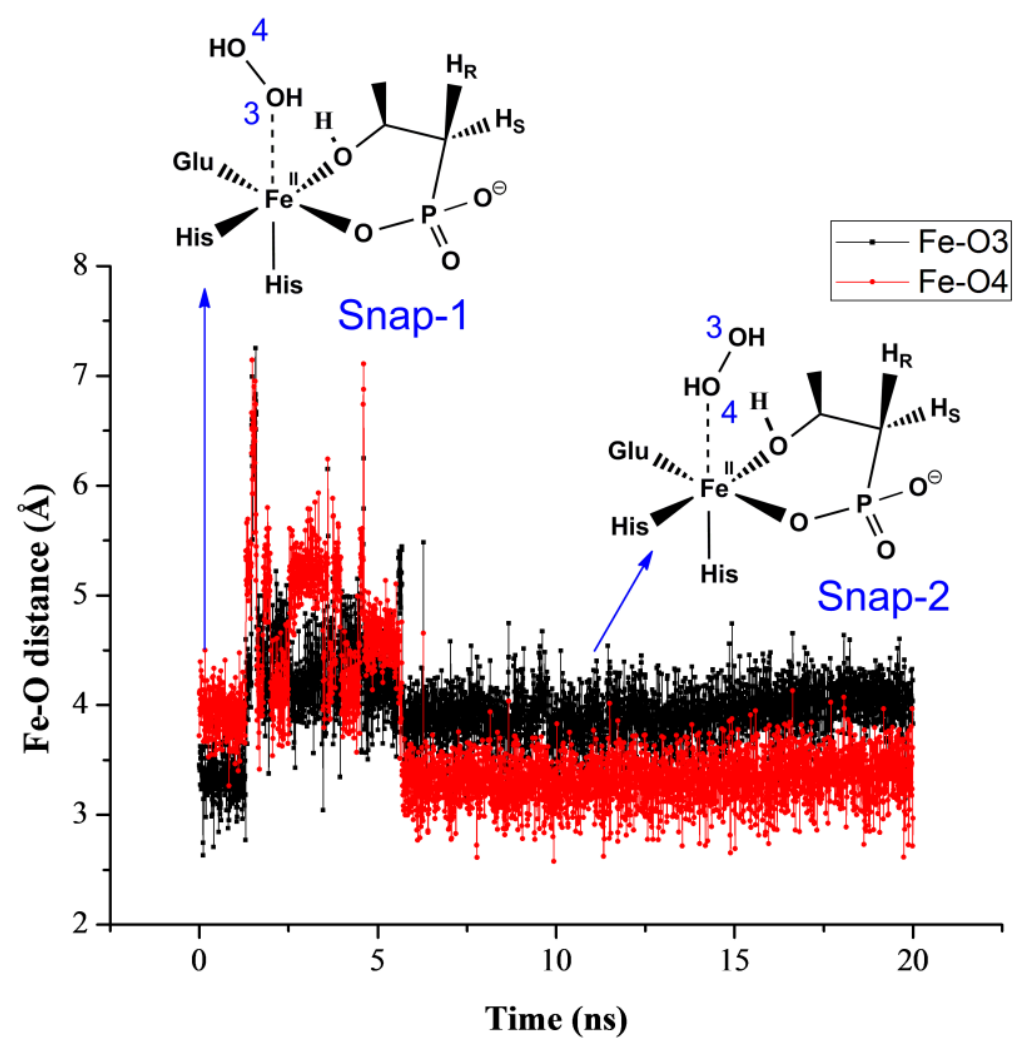

Figure S1. Fe-O3 and Fe-O4 distance fluctuations during $20 \mathrm{~ns}$ of relaxed MD simulations of $\mathrm{Fe}(\mathrm{II})-\mathrm{H}_{2} \mathrm{O}_{2}$. MD shows a clear overturn of $\mathrm{H}_{2} \mathrm{O}_{2}$ molecular. 


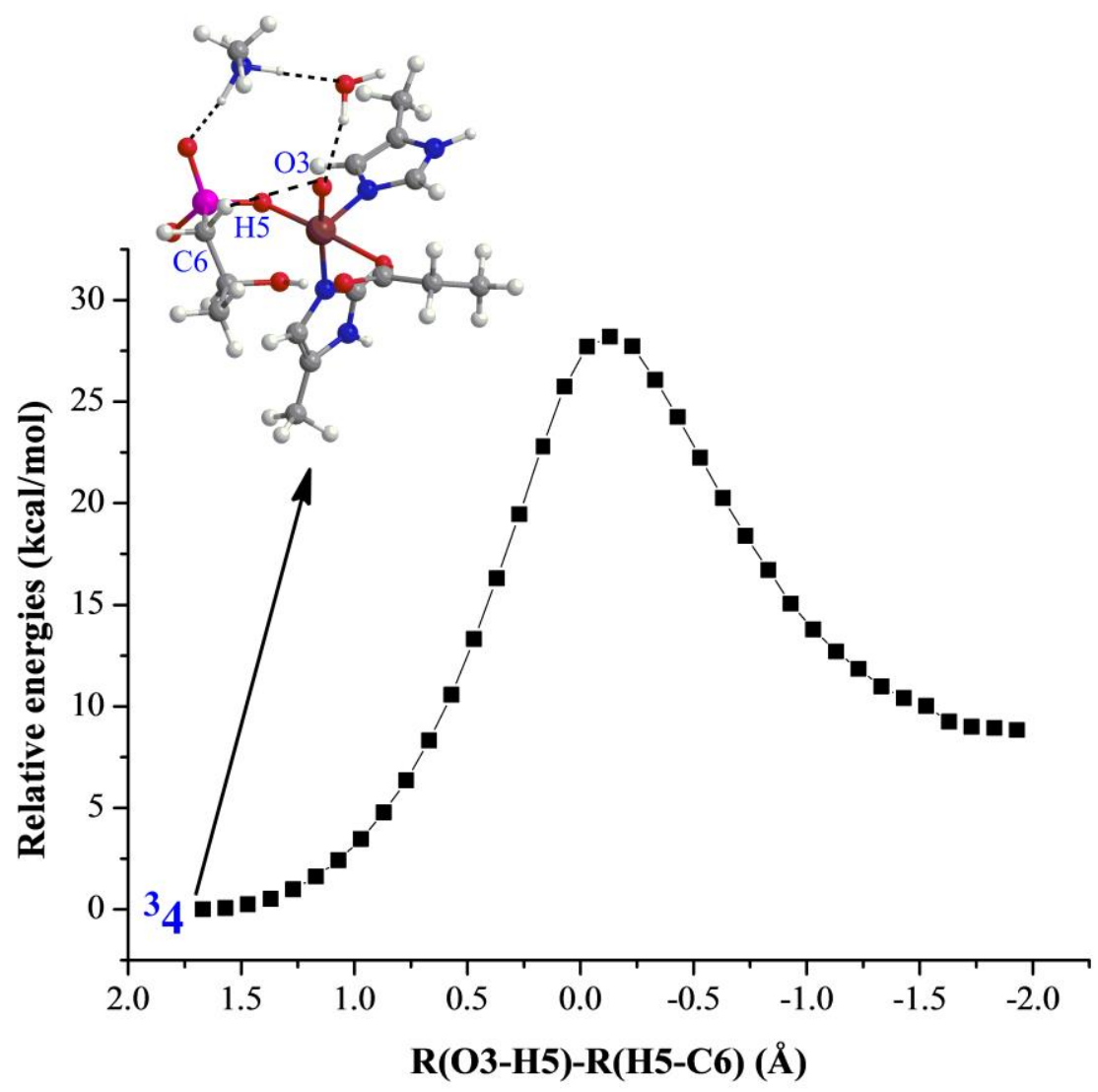

Figure S2. Scanned energy surface for the $H_{R}$-abstraction by triplet $\mathrm{Fe}(\mathrm{IV})=\mathrm{O}$ species. 


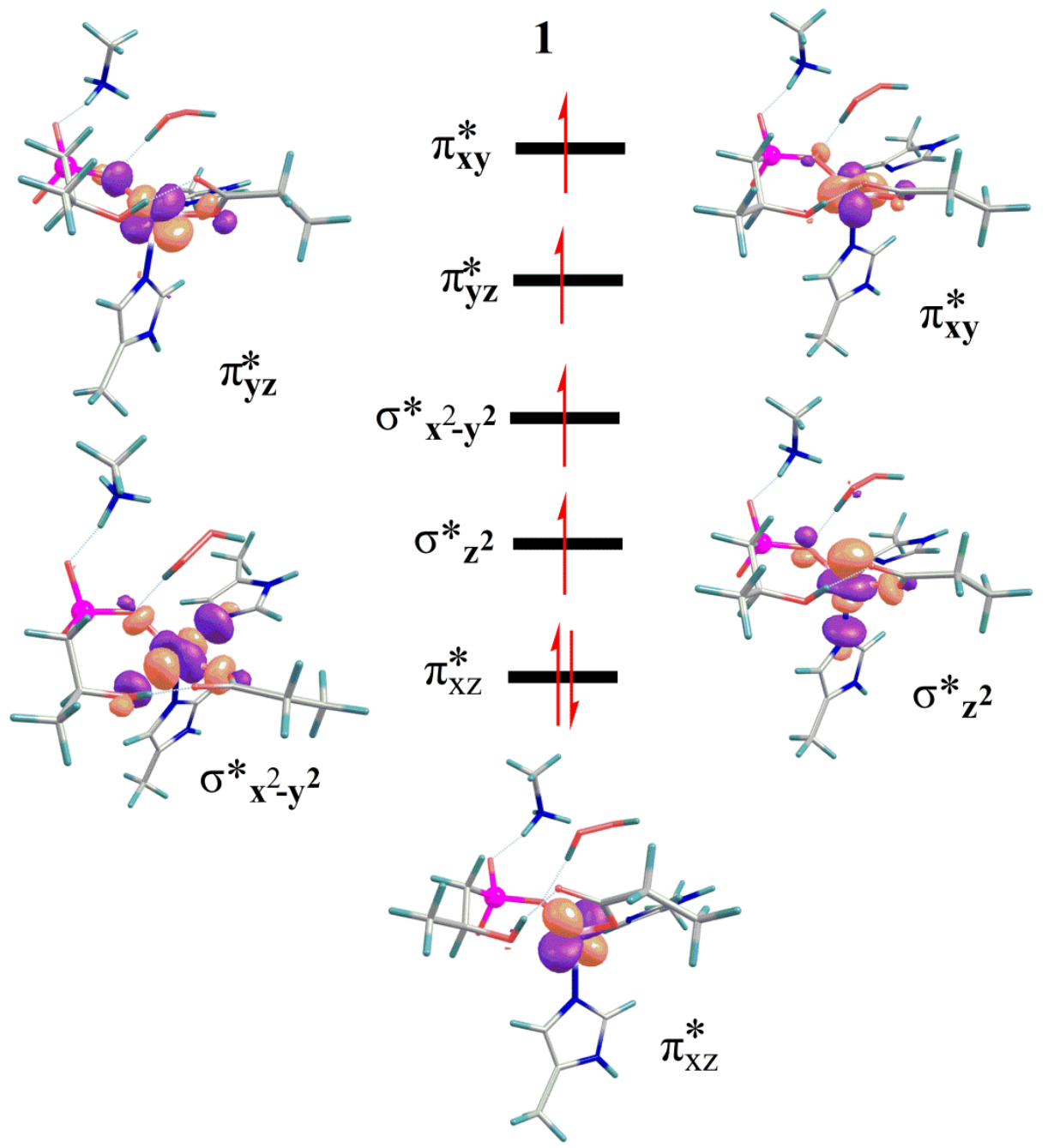

Figure S3. Spin natural orbital $(\mathrm{SNO})$ of $\mathbf{1}, \mathrm{Fe}^{\mathrm{II}}-\mathrm{H}_{2} \mathrm{O}_{2}$ reactant complex. 

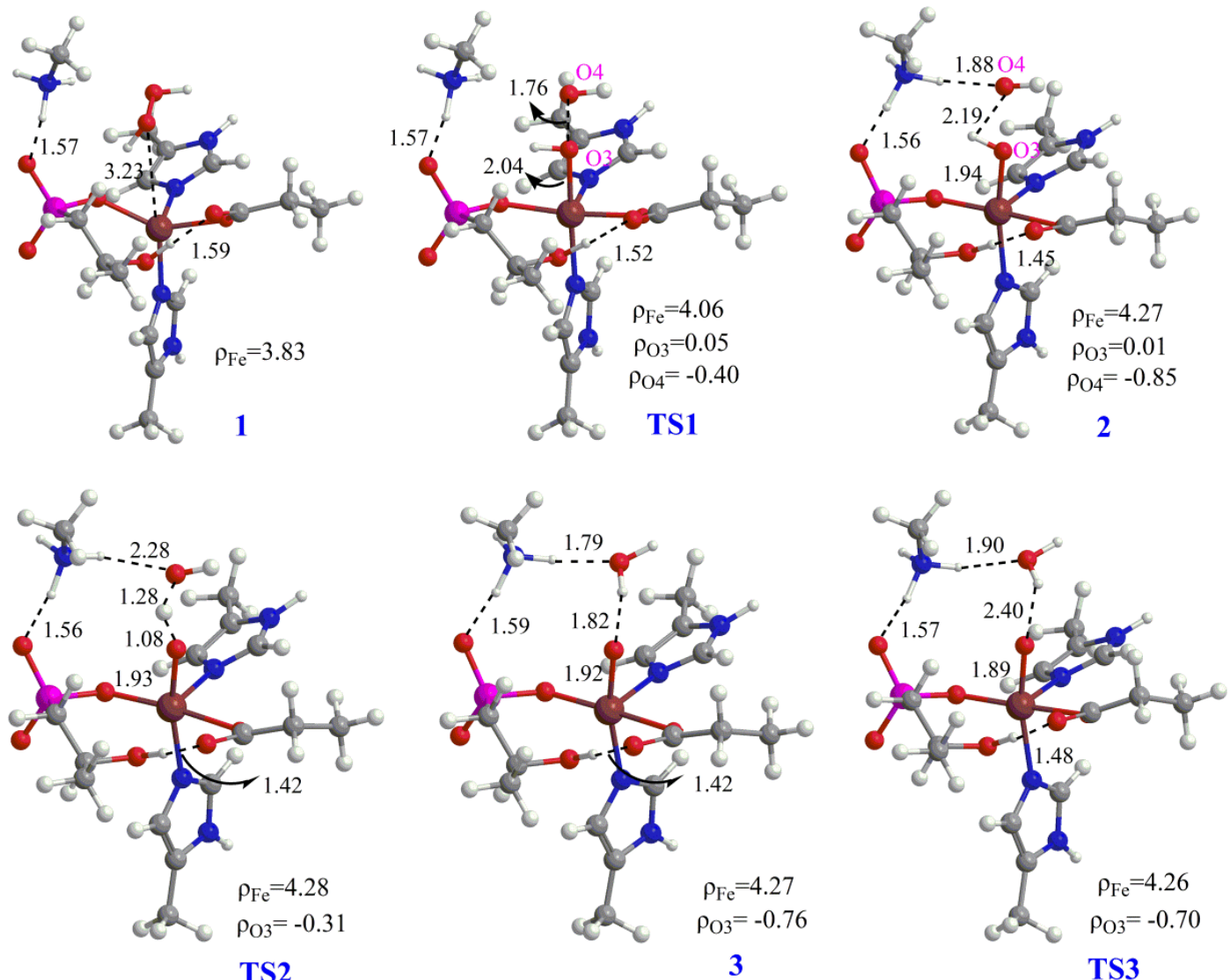

TS2
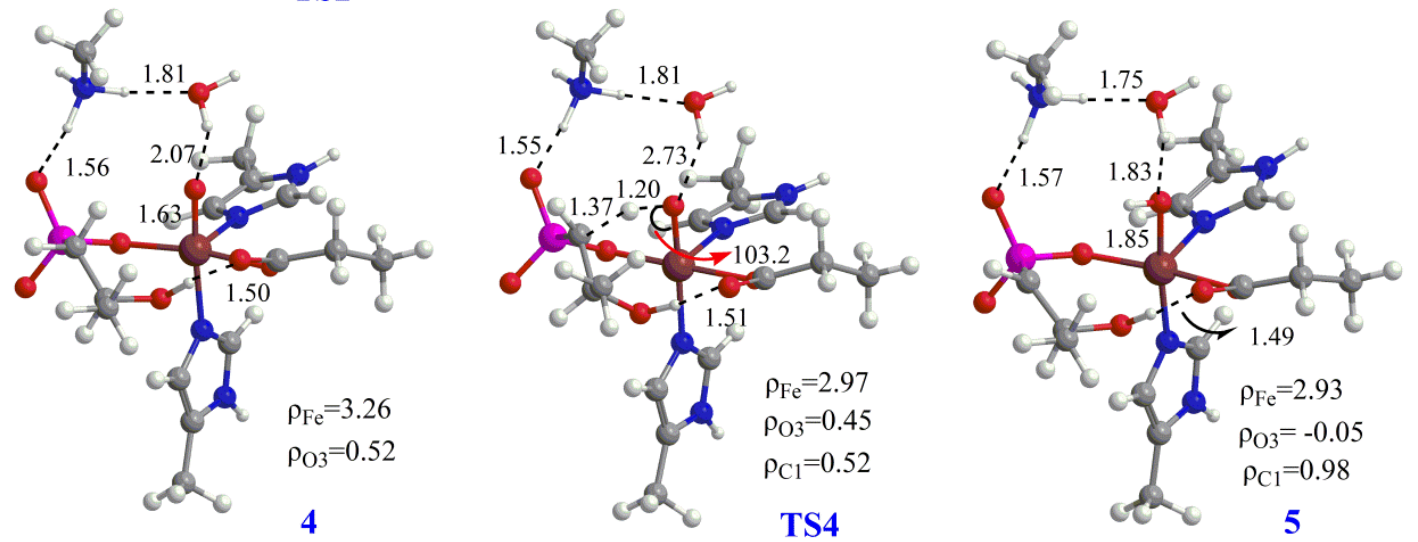

Figure S4. QM/MM (UB3LYP/B1) optimized structures (in $\AA$ ) involved in $\mathrm{Fe}^{\mathrm{III}}-\mathrm{O} \cdot / \mathrm{Fe}^{\mathrm{IV}}=\mathrm{O}$ formation and $\mathrm{H}$-abstraction by $\mathrm{Fe}^{\mathrm{IV}}=\mathrm{O}$ species. The spin densities on key atoms are also shown along with the structures. 


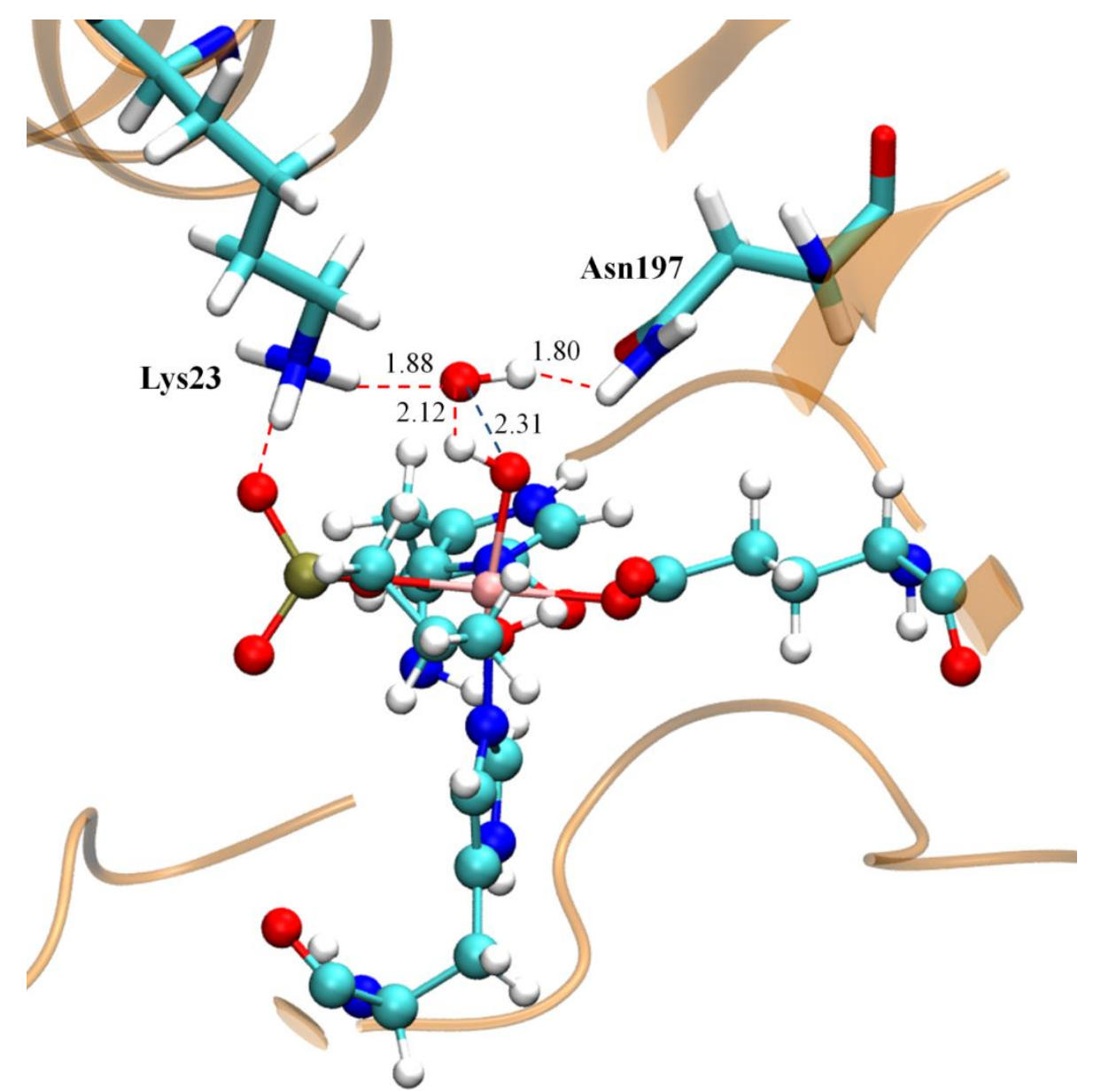

Figure S5. QM/MM (UB3LYP/B1) optimized structures (distances in $\AA$ ) of $\mathrm{Fe}^{\mathrm{III}}-\mathrm{OH} / \mathrm{HO} \cdot$ intermediate of 2.

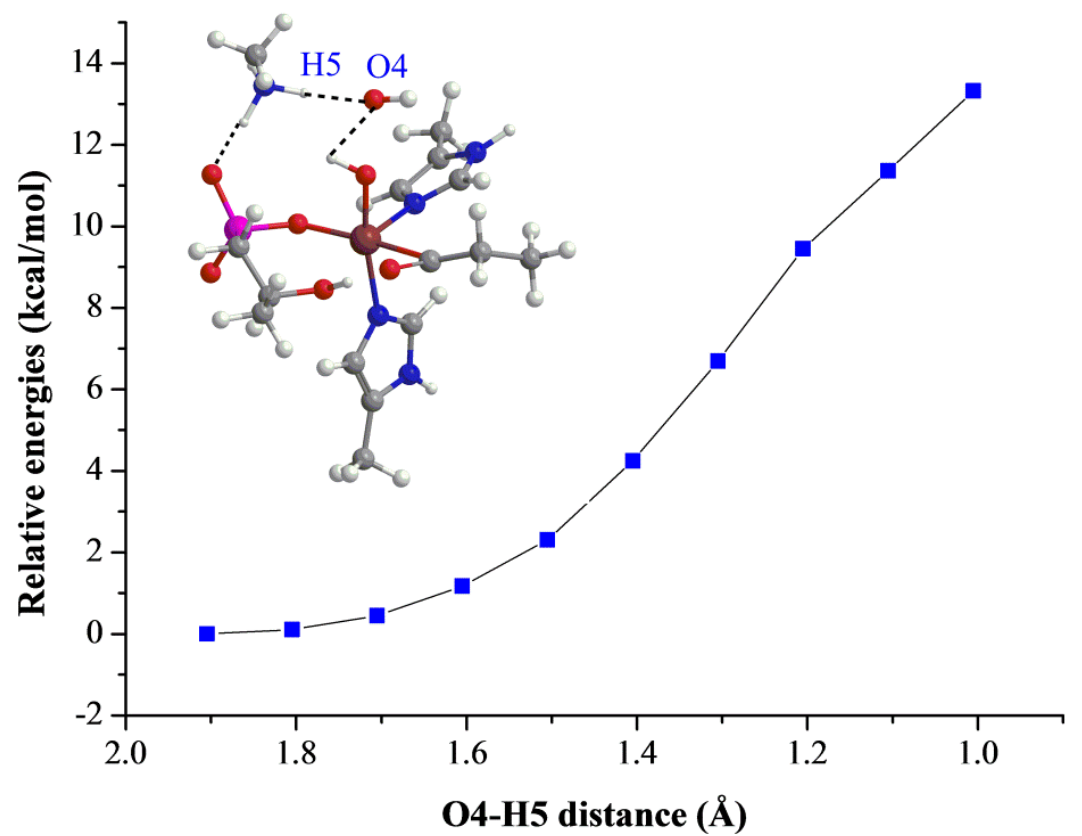

Figure S6. Scanned energy surface for the $\mathrm{H}$-abstraction from Lys 23 by $\mathrm{HO} \bullet$ radical in 2. 

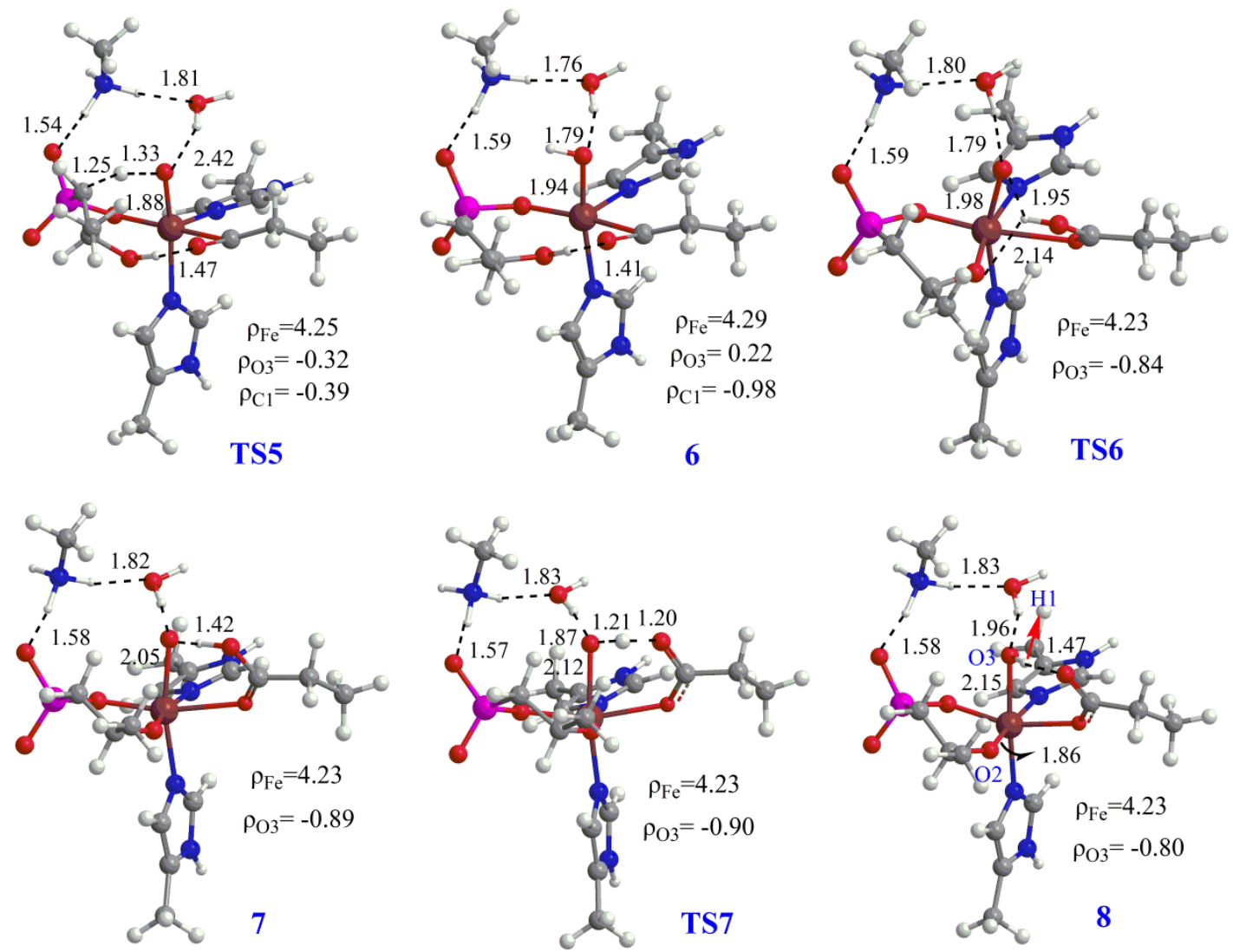

Figure S7. QM/MM (UB3LYP/B1) optimized structures (distances in $\AA$ ) involved in $\mathrm{H}$-abstraction and $\mathrm{Fe}^{\mathrm{III}}-(\mathrm{HO} \bullet)$ formation starting from $\mathrm{Fe}^{\mathrm{III}}-\mathrm{O} \bullet$ species of 4 . The spin density $(\rho)$ at key atoms are also shown along with the structures.

(a)

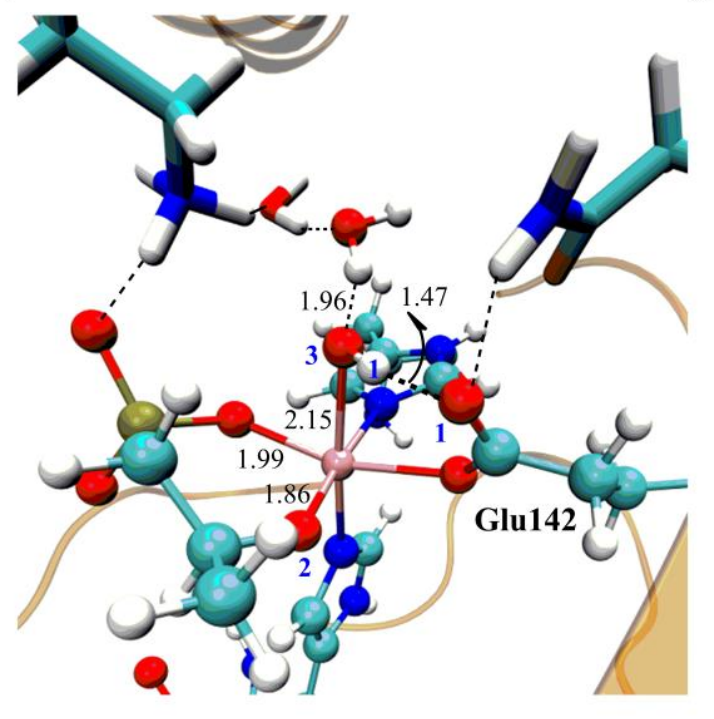

(b)

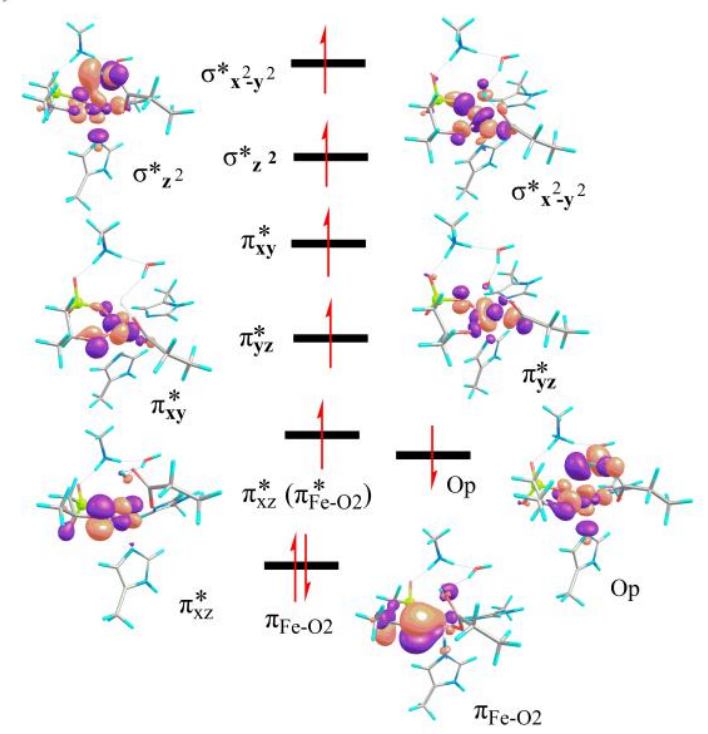

Figure S8. (a) Key geometric feature of the $\mathrm{Fe}^{\mathrm{III}}-(\mathrm{HO} \cdot)$ species $(\mathbf{8})$, showing the 
$\mathrm{Fe}-\mathrm{O} 2$ and $\mathrm{Fe}-\mathrm{O} 3$ distances, as well as the $\mathrm{H}$-bonding interaction between $\mathrm{H} 1$ and $\mathrm{O} 1$ of Glu142. (b) Spin natural orbitals of $\mathrm{Fe}^{\mathrm{III}}-(\mathrm{HO} \cdot)$. Note the antibonding interaction $\mathrm{Fe}-\mathrm{O} 2$ in the $\pi^{*}$ d-type orbitals, and the strong bonding in $\pi_{\mathrm{Fe}-\mathrm{O} 2}$.
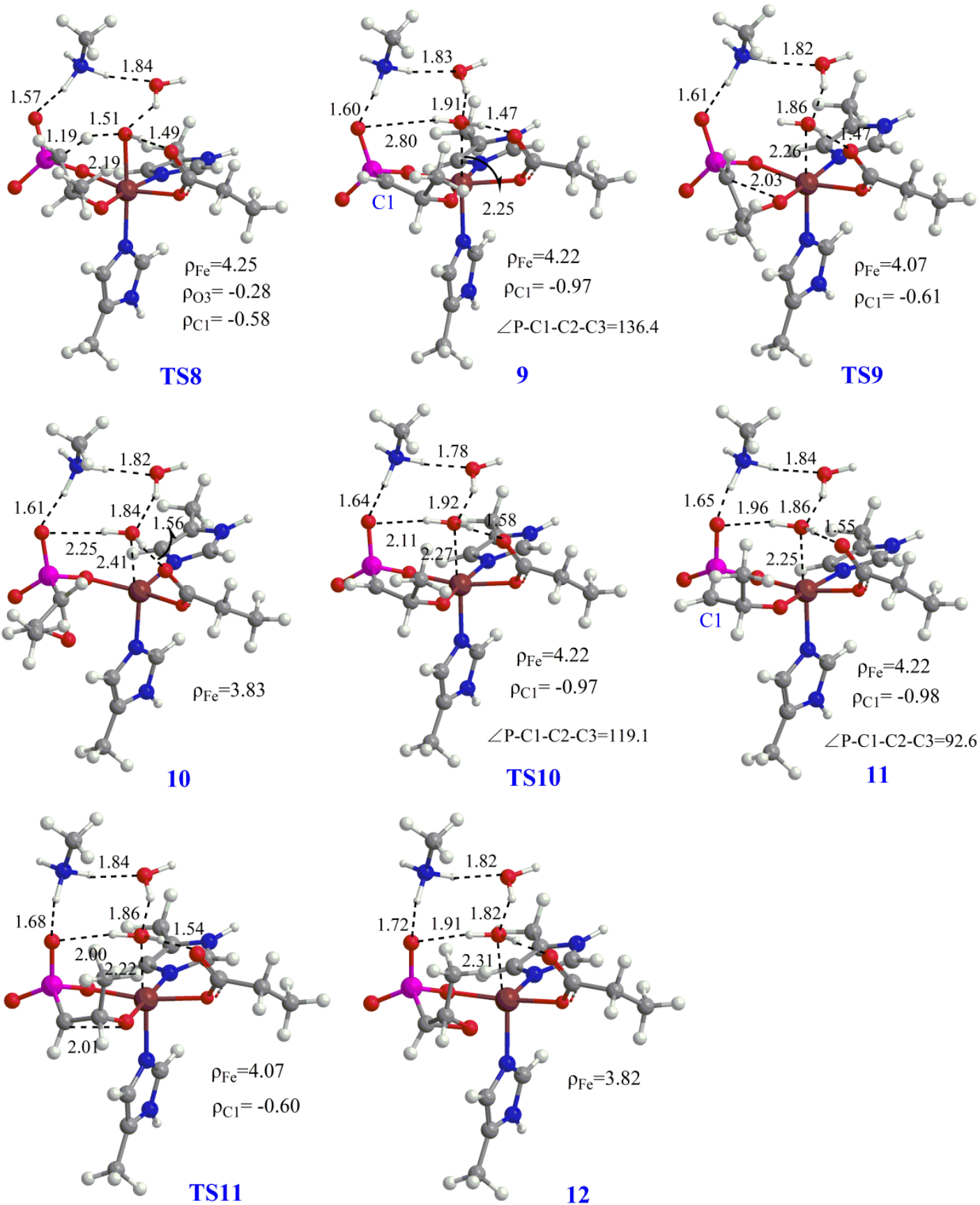

Figure S9. QM/MM (UB3LYP/B1) optimized structures (distances in $\AA$, angles in degrees) involved in $\mathrm{C}-\mathrm{H}$ activation and fosfomycin formation starting from $\mathrm{Fe}^{\mathrm{III}}-(\mathrm{HO} \cdot)$ species of $\mathbf{8}$. The spin densities $(\rho)$ at key atoms are also shown along with the structures. 

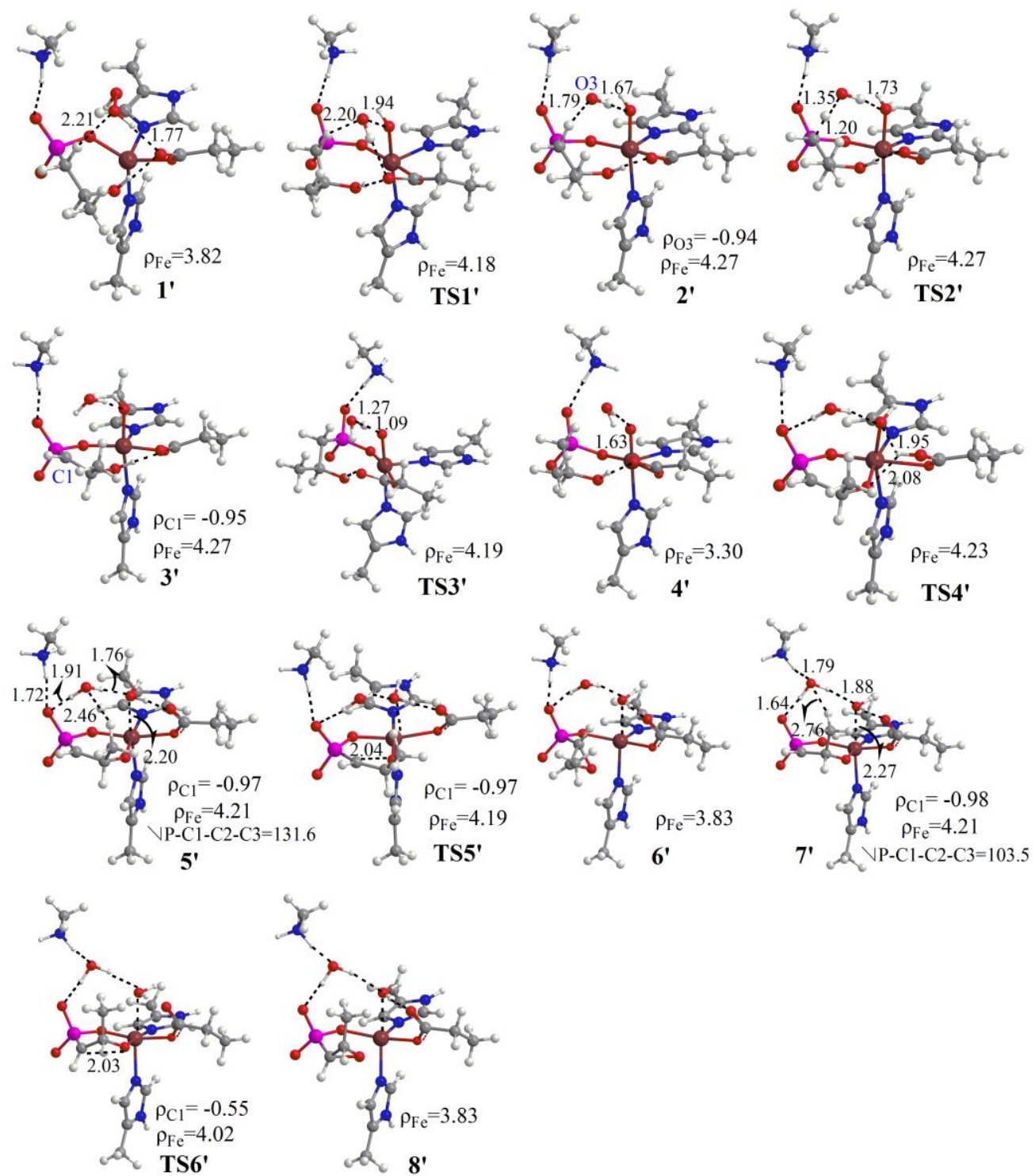

Figure S10. QM/MM (UB3LYP/B1) optimized structures (distances in $\AA$, angles in degrees) involved in fosfomycin formation starting from $\mathbf{1}^{\prime}$ in Snap-2. The spin densities $(\rho)$ at key atoms are also shown along with the structures.

Conformation Change $5^{\prime}$ to $7^{\prime}$ : We did not locate the direct transformation by scanning the dihedral $\angle \mathrm{P}-\mathrm{C} 1-\mathrm{C} 2-\mathrm{C} 3$. Then we fixed the Fe-ligating residues and performed a short MD run (100ps), and the QM/MM optimization of a snapshot taken from this short MD will automatically leads to $7^{\prime}$. Clearly, the conformation change of $5^{\prime}$ to $7^{\prime}$ is driven by the dynamic of surrounding residues in the active site (mostly the change of H-bond networks as shown in Figure S10), and such dynamic process is quite fast. 
Zero-point energies (ZPE) at B1, total QM/MM energies at B2, as well as Cartesian coordinates, of all computed species

\section{1}

ZPE at B1: 0.493987 (a.u.)

Total QM/MM energy at B2: -3166.523718 (a.u.)

$\mathrm{Fe} \quad 2.051118-11.89425819 .841093$

P $\quad-0.343934-10.49516321 .624888$

C $\quad 0.910674-10.33462322 .943400$

C $\quad 1.685614-11.61525223 .260803$

O $2.434628-12.05983622 .128285$

C $\quad 2.588351-11.43681224 .481056$

O $\quad-1.300355-11.64924321 .953966$

O $\quad 0.440697-10.73719120 .246101$

O $\quad-1.025829-9.09698921 .516953$

$\mathrm{H} \quad 0.364984-9.99672423 .841607$

H $\quad 1.994902-11.16094725 .367449$

H $\quad 3.135164-12.36251524 .711398$

H $\quad 3.330279-10.64177624 .298145$

H $\quad 0.946024-12.40914823 .466405$

C $\quad-0.107931-10.86524514 .544283$

$\mathrm{H} \quad 0.061673-9.91317514 .015710$

H $\quad-1.122837-10.81000014 .965504$

$\mathrm{N} \quad 2.216724-10.80915115 .611634$

H $\quad 2.730191-10.49526214 .777434$

C $\quad 0.857361-11.05796615 .674887$

C $\quad 2.753603-11.06504416 .823834$

H $3.813194-10.95300917 .039526$

N $\quad 1.821542-11.47015617 .677381$

C $\quad 0.637099-11.46858916 .972046$

H $\quad-0.307875-11.74480717 .434066$

C $\quad 1.114147-17.47322120 .952000$

H $\quad 1.579975-17.49027321 .948982$

H $\quad 1.613720-18.25388520 .355645$

$\mathrm{N} \quad 1.391149-15.96695418 .924302$

H $\quad 1.231275-16.66451718 .180018$

C $\quad 1.314344-16.13951020 .296676$

C $\quad 1.629490-14.67072618 .658219$

$\mathrm{H} \quad 1.755538-14.27802817 .651421$

N $\quad 1.719950-13.97761919 .783819$

C $\quad 1.533429-14.87974120 .814537$

H $\quad 1.601871-14.56590521 .853456$

C $\quad 6.739327-11.86670518 .703520$

$\mathrm{H} \quad 6.085883-11.53192417 .881651$
$6.585779-12.95029818 .825189$

C $\quad 6.388440-11.10461119 .976242$

H $7.005332-11.40438320 .839313$

H $\quad 6.568816-10.02068719 .855513$

C $\quad 4.930291-11.24926920 .417897$

O $\quad 4.627926-10.83324521 .551508$

O $4.099299-11.78335219 .594069$

O $\quad 2.118264-8.67701619 .547844$

H $\quad 1.610935-9.52993022 .660014$

H $\quad 3.294481-11.53621622 .050479$

O $\quad 1.909301-8.26919118 .180188$

H $\quad 2.816380-8.34205517 .806487$

H $\quad 1.432634-9.38624519 .672909$

C $\quad 0.030984-6.20775120 .159922$

H $\quad 0.381929-5.56184519 .344983$

H $\quad 0.900132-6.64882520 .660108$

$\mathrm{N} \quad-0.776044-7.32246619 .585546$

H $\quad-0.430966-7.63962118 .660411$

H $\quad-0.824679-8.13801320 .286141$

H $\quad-1.759008-7.03796419 .415595$

h $\quad-0.156563-11.69757413 .842192$

h $\quad 0.078561-17.80744921 .014609$

h $\quad 7.744469-11.72583818 .306137$

h $\quad-0.575392-5.61210420 .842255$

\section{TS1}

ZPE at B1: 0.480683 (a.u.)

Total QM/MM energy at B2: -3166.496695 (a.u.)

Fe $\quad 2.032017-11.50938019 .750857$

P $\quad-0.526693-10.57782621 .652539$

C $\quad 0.772291-10.37671222 .927687$

C $\quad 1.664268-11.59769423 .138308$

O $2.404437-11.89146821 .952432$

C $\quad 2.592856-11.42535824 .338615$

O $\quad-1.485628-11.69584322 .071749$

O $\quad 0.177368-10.90619220 .266988$

O $\quad-1.169243-9.15785221 .528489$

H $\quad 0.235288-10.13264323 .860642$

H $\quad 2.007914-11.25910725 .256938$

H $\quad 3.217719-12.31683224 .491252$

H $\quad 3.264230-10.56341624 .190736$ 


\begin{tabular}{|c|c|c|c|}
\hline $\mathrm{H}$ & $1.001098-12.46454423 .317749$ & $\mathrm{H}$ & $-1.777145-7.09129619 .405257$ \\
\hline $\mathrm{C}$ & $-0.149340-10.86091314 .454736$ & $\mathrm{~h}$ & $-0.179587-11.70319513 .763568$ \\
\hline $\mathrm{H}$ & $0.003022-9.91216113 .915200$ & $\mathrm{~h}$ & $0.212002-17.61945520 .996852$ \\
\hline $\mathrm{H}$ & $-1.165608-10.82102214 .873862$ & $\mathrm{~h}$ & $7.785397-11.73969418 .266117$ \\
\hline $\mathrm{N}$ & $2.178940-10.78148515 .511741$ & $\mathrm{~h}$ & $-0.607803-5.65381420 .843761$ \\
\hline $\mathrm{H}$ & $2.687518-10.48161414 .668349$ & & \\
\hline $\mathrm{C}$ & $0.817495-11.02047315 .588200$ & 2 & \\
\hline $\mathrm{C}$ & $2.723356-11.00499016 .723890$ & $\mathrm{ZPI}$ & at B1: 0.485526 (a.u.) \\
\hline $\mathrm{H}$ & $3.784408-10.88782916 .931918$ & Tot & QM/MM energy at B2: -3166.512359 (a.u.) \\
\hline $\mathrm{N}$ & $1.790634-11.37888017 .590746$ & $\mathrm{Fe}$ & $2.079997-11.38433719 .801314$ \\
\hline $\mathrm{C}$ & $0.599273-11.38850416 .897438$ & $\mathrm{P}$ & $-0.463134-10.55101121 .697531$ \\
\hline $\mathrm{H}$ & $-0.342893-11.63981017 .378311$ & $\mathrm{C}$ & $0.844523-10.36462922 .958477$ \\
\hline $\mathrm{C}$ & $1.213113-17.19607220 .915677$ & $\mathrm{C}$ & $1.736729-11.59058923 .117407$ \\
\hline $\mathrm{H}$ & $1.687430-17.16308721 .908423$ & $\mathrm{O}$ & $2.453504-11.84229421 .903471$ \\
\hline $\mathrm{H}$ & $1.769653-17.94516920 .327707$ & $\mathrm{C}$ & $2.690482-11.45576324 .301450$ \\
\hline $\mathrm{N}$ & $1.384684-15.69808018 .864849$ & $\mathrm{O}$ & $-1.424823-11.66916322 .101575$ \\
\hline $\mathrm{H}$ & $1.240986-16.41319418 .133591$ & $\mathrm{O}$ & $0.257197-10.90500920 .313944$ \\
\hline $\mathrm{C}$ & $1.329104-15.85924920 .240377$ & $\mathrm{O}$ & $-1.102077-9.13737621 .561856$ \\
\hline $\mathrm{C}$ & $1.613408-14.39991418 .589137$ & $\mathrm{H}$ & $0.321931-10.14611823 .905346$ \\
\hline $\mathrm{H}$ & $1.726557-14.01778117 .577737$ & $\mathrm{H}$ & $2.119750-11.30950925 .231789$ \\
\hline $\mathrm{N}$ & $1.714337-13.69277819 .704948$ & $\mathrm{H}$ & $3.308697-12.35607824 .423185$ \\
\hline $\mathrm{C}$ & $1.543951-14.59059120 .742289$ & $\mathrm{H}$ & $3.365904-10.59566724 .165161$ \\
\hline $\mathrm{H}$ & $1.628431-14.27302721 .777725$ & $\mathrm{H}$ & $1.076346-12.46211823 .285744$ \\
\hline $\mathrm{C}$ & $6.777330-11.88749118 .653445$ & $\mathrm{C}$ & $-0.143725-10.85112814 .517322$ \\
\hline $\mathrm{H}$ & $6.126885-11.52745317 .839939$ & $\mathrm{H}$ & $0.009006-9.90412613 .974850$ \\
\hline $\mathrm{H}$ & $6.623342-12.97392918 .746661$ & $\mathrm{H}$ & $-1.159129-10.80329314 .937865$ \\
\hline $\mathrm{C}$ & $6.431902-11.15768419 .943706$ & $\mathrm{~N}$ & $2.182511-10.74019515 .574517$ \\
\hline $\mathrm{H}$ & $7.056428-11.47148420 .795460$ & $\mathrm{H}$ & $2.686701-10.43792614 .728346$ \\
\hline $\mathrm{H}$ & $6.606048-10.07040619 .841533$ & $\mathrm{C}$ & $0.824558-11.00047715 .651576$ \\
\hline $\mathrm{C}$ & $4.979442-11.30797620 .395849$ & $\mathrm{C}$ & $2.729254-10.93514716 .787686$ \\
\hline $\mathrm{O}$ & $4.723271-10.98349021 .571522$ & $\mathrm{H}$ & $3.784364-10.78276417 .000108$ \\
\hline $\mathrm{O}$ & $4.109343-11.73514019 .550584$ & $\mathrm{~N}$ & $1.801454-11.32116817 .656471$ \\
\hline $\mathrm{O}$ & $2.416646-9.51192319 .843232$ & $\mathrm{C}$ & $0.609448-11.36024716 .963666$ \\
\hline $\mathrm{H}$ & $1.396990-9.50257422 .673735$ & $\mathrm{H}$ & $-0.327837-11.62886317 .443821$ \\
\hline $\mathrm{H}$ & $3.341720-11.48540921 .961321$ & $\mathrm{C}$ & $1.249626-17.09409220 .885802$ \\
\hline $\mathrm{O}$ & $2.127851-8.41017718 .498476$ & $\mathrm{H}$ & $1.731057-17.04216821 .874373$ \\
\hline $\mathrm{H}$ & $3.020635-8.50317218 .096459$ & $\mathrm{H}$ & $1.822060-17.83188920 .298594$ \\
\hline $\mathrm{H}$ & $1.569647-9.24658120 .236642$ & $\mathrm{~N}$ & $1.388696-15.60287618 .824835$ \\
\hline $\mathrm{C}$ & $0.000314-6.27440920 .185641$ & $\mathrm{H}$ & $1.253581-16.32582518 .098657$ \\
\hline $\mathrm{H}$ & $0.384268-5.64926219 .369111$ & $\mathrm{C}$ & $1.332876-15.75867120 .200867$ \\
\hline $\mathrm{H}$ & $0.853654-6.70425120 .724698$ & $\mathrm{C}$ & $1.609208-14.30451618 .542951$ \\
\hline $\mathrm{N}$ & $-0.804036-7.38933119 .608650$ & $\mathrm{H}$ & $1.724483-13.92838917 .530000$ \\
\hline $\mathrm{H}$ & $-0.428087-7.72497418 .694796$ & $\mathrm{~N}$ & $1.702368-13.59068519 .655747$ \\
\hline $\mathrm{H}$ & $-0.899618-8.19964220 .314505$ & $\mathrm{C}$ & $1.535477-14.48584520 .696847$ \\
\hline
\end{tabular}




$$
\begin{array}{ll}
\mathrm{H} & 1.617097-14.16692521 .731506 \\
\mathrm{C} & 6.804037-11.95559418 .630605 \\
\mathrm{H} & 6.143431-11.59067617 .827424 \\
\mathrm{H} & 6.668600-13.04564718 .703732 \\
\mathrm{C} & 6.467886-11.25513419 .938233 \\
\mathrm{H} & 7.117590-11.56378120 .772437 \\
\mathrm{H} & 6.611986-10.16268919 .847278 \\
\mathrm{C} & 5.033910-11.43814720 .420242 \\
\mathrm{O} & 4.815095-11.17760221 .621206 \\
\mathrm{O} & 4.132530-11.81855219 .586331 \\
\mathrm{O} & 2.680468-9.53637719 .802600 \\
\mathrm{H} & 1.463043-9.48631122 .706826 \\
\mathrm{H} & 3.451198-11.54508921 .938065 \\
\mathrm{O} & 1.755903-8.24135018 .125680 \\
\mathrm{H} & 2.686193-8.26566817 .808259 \\
\mathrm{H} & 1.998318-8.97696520 .196698 \\
\mathrm{C} & 0.136107-6.25868320 .251892 \\
\mathrm{H} & 0.579074-5.64113419 .458878 \\
\mathrm{H} & 0.944594-6.63727820 .891268 \\
\mathrm{~N} & -0.532177-7.43088919 .632553 \\
\mathrm{H} & 0.113947-7.86630618 .951064 \\
\mathrm{H} & -0.786525-8.16506520 .377081 \\
\mathrm{H} & -1.396419-7.18474419 .126633 \\
\mathrm{~h} & -0.178203-11.69252313 .825273 \\
\mathrm{~h} & 0.262593-17.54683320 .979919 \\
& 7.808384-11.78555718 .242737 \\
\mathrm{H} & -0.537581-5.64135620 .846130
\end{array}
$$

\section{TS2}

ZPE at B1: 0.483877 (a.u.)

Total QM/MM energy at B2: -3166.507790 (a.u.)

$$
\mathrm{Fe} \quad 2.080152-11.39785319 .816299
$$$$
\text { P } \quad-0.480818-10.55886821 .630398
$$$$
\text { C } \quad 0.834603-10.31266622 .870962
$$$$
\text { C } \quad 1.720691-11.53168623 .093487
$$$$
\text { O } 2.434469-11.84083821 .889373
$$$$
\text { C } \quad 2.680791-11.34281024 .265183
$$$$
\text { O } \quad-1.425454-11.67954122 .071642
$$$$
\text { O } \quad 0.233438-10.94247720 .250178
$$$$
\text { O } \quad-1.151029-9.16255921 .472912
$$$$
\text { H } \quad 0.320147-10.03729823 .807489
$$$$
\text { H } \quad 2.114174-11.14915025 .189425
$$$$
\text { H } \quad 3.295442-12.23946324 .428595
$$

H $\quad 3.358797-10.49367324 .083352$
$\mathrm{H}$

H $\quad-1.125102-10.80933114 .954997$

N $\quad 2.217528-10.74446315 .583515$

$\mathrm{H}$

C $\quad 0.859694-11.00938915 .659389$

C $\quad 2.761770-10.92742716 .800066$

H $\quad 3.815675-10.77002917 .014408$

N $\quad 1.831880-11.30699717 .669526$

C $\quad 0.642092-11.35707116 .973438$

H $\quad-0.295281-11.61799117 .456849$

C $\quad 1.246304-17.10136320 .881057$

H $\quad 1.729966-17.04913921 .868514$

H $\quad 1.816232-17.84030720 .292886$

$\mathrm{N} \quad 1.383206-15.61204518 .818904$

H $\quad 1.248825-16.33586018 .093756$

C $\quad 1.329034-15.76644520 .195300$

C $\quad 1.600990-14.31323318 .535845$

H $\quad 1.714884-13.93729917 .522667$

N $\quad 1.693693-13.59884419 .647632$

C $\quad 1.529398-14.49288720 .690097$

H $\quad 1.610358-14.17014721 .723768$

C $\quad 6.810776-12.01353118 .632412$

H $\quad 6.139783-11.60230317 .861051$

H $\quad 6.680262-13.10650218 .636426$

C $\quad 6.486303-11.40263819 .986061$

H $\quad 7.123441-11.78978320 .796838$

H $\quad 6.662015-10.31131919 .981256$

C $\quad 5.045117-11.57803820 .449815$

O $\quad 4.822192-11.32118121 .654393$

O $4.147174-11.94221619 .612777$

O $\quad 2.777644-9.60121719 .853254$

H $\quad 1.454057-9.45730522 .552543$

H $\quad 3.461107-11.61216621 .932557$

O $\quad 1.887902-8.20951318 .323989$

H $\quad 2.748699-8.14510617 .851666$

H $\quad 2.217959-8.77909219 .420574$

C $\quad 0.040715-6.25638820 .188035$

$\mathrm{H} \quad 0.441044-5.62681519 .382849$

H $\quad 0.881055-6.68386620 .748527$

N $\quad-0.735074-7.37737919 .590160$

$\mathrm{H} \quad-0.304922-7.73053318 .716786$

H $\quad-0.844859-8.18171920 .296383$ 
H $\quad-1.698816-7.09291819 .336867$

h $\quad-0.154301-11.69615613 .834565$

h $\quad 0.258610-17.55216920 .977496$

h $\quad 7.810901-11.82058418 .244355$

h $\quad-0.584693-5.64151620 .835231$

\section{3}

ZPE at B1: 0.487400 (a.u.)

Total QM/MM energy at B2: -3166.530558 (a.u.)

$\mathrm{Fe} \quad 2.068082-11.40402619 .852760$

$\mathrm{P} \quad-0.460101-10.55617921 .723927$

C $\quad 0.860808-10.34530022 .963815$

C $\quad 1.766353-11.55955623 .135621$

O $2.480193-11.82892921 .924945$

C $\quad 2.723204-11.38619424 .311908$

O $\quad-1.412269-11.67667722 .145918$

O $\quad 0.250857-10.94179020 .341473$

O $\quad-1.112782-9.15379321 .569119$

H $\quad 0.345455-10.12356523 .913714$

H $\quad 2.155085-11.23241925 .242482$

H $\quad 3.361059-12.27048524 .448482$

H $\quad 3.377735-10.51278224 .159095$

H $\quad 1.117636-12.43553323 .324891$

C $\quad-0.134385-10.88783914 .557886$

H $\quad 0.018869-9.92592714 .041928$

H $\quad-1.147393-10.84492814 .984011$

N $\quad 2.203708-10.86399215 .594949$

H $\quad 2.714974-10.58856714 .747884$

C $\quad 0.836990-11.06772915 .684474$

C $\quad 2.749211-11.02402016 .814238$

H $\quad 3.809917-10.88916617 .017716$

N $\quad 1.810014-11.35006017 .696076$

C $\quad 0.614960-11.37539417 .007548$

H $\quad-0.329148-11.58819417 .501421$

C $\quad 1.269222-17.10052020 .903464$

H $1.747829-17.05049221 .893468$

H $\quad 1.842498-17.83727820 .315866$

N $\quad 1.413722-15.60560918 .845901$

H $\quad 1.280700-16.32660018 .117399$

C $\quad 1.353449-15.76387220 .221685$

C $\quad 1.631398-14.30679718 .566563$

H $\quad 1.747312-13.92872717 .554679$

N $\quad 1.718052-13.59474619 .681245$

C $\quad 1.549413-14.49165320 .720825$
$\mathrm{H}$

C $\quad 6.826211-11.90541618 .639220$

H $\quad 6.169634-11.50687017 .848815$

H $\quad 6.674678-12.99527718 .686490$

C $\quad 6.495970-11.23409619 .964489$

H $\quad 7.138615-11.57169420 .792993$

H $\quad 6.649197-10.14063319 .901593$

C $\quad 5.058618-11.41904820 .437317$

O $4.835214-11.18008421 .643827$

O $\quad 4.158868-11.78549819 .597379$

O $\quad 2.685573-9.58835419 .757360$

H $\quad 1.463904-9.46195822 .693034$

H $\quad 3.486910-11.51726921 .942714$

O $\quad 1.356372-8.14071017 .775576$

H $\quad 1.897531-7.92494416 .983044$

H $\quad 1.949294-8.62511118 .399161$

C $\quad 0.097026-6.26032720 .249425$

H $\quad 0.532673-5.64494719 .450472$

H $\quad 0.911561-6.66348220 .865902$

N $\quad-0.609943-7.41255419 .631859$

H $\quad-0.031013-7.78658918 .850894$

H $\quad-0.783235-8.17780120 .361415$

H $\quad-1.534084-7.15577219 .246582$

h $\quad-0.170659-11.71180813 .845267$

h $\quad 0.280935-17.55142320 .993137$

h $\quad 7.831726-11.74504018 .250266$

h $\quad-0.558417-5.63854020 .859213$

TS3

ZPE at B1: 0.486764 (a.u.)

Total QM/MM energy at B2: -3166.528838 (a.u.)

$\mathrm{Fe} \quad 2.087551-11.49516619 .925712$

P $\quad-0.424418-10.57114421 .738624$

C $\quad 0.913884-10.35473122 .960460$

C $\quad 1.741067-11.60932523 .224247$

O $\quad 2.429758-12.01494122 .032218$

C $\quad 2.716091-11.41671624 .383296$

O $\quad-1.392959-11.66598522 .190288$

O $\quad 0.258859-10.98871520 .352933$

O $\quad-1.057759-9.15894721 .562751$

H $\quad 0.420782-10.02633123 .891369$

H $\quad 2.165750-11.14922825 .298940$

H $\quad 3.283028-12.33603624 .588589$

H $\quad 3.436944-10.61475024 .157746$ 


\begin{tabular}{|c|c|c|c|}
\hline $\mathrm{H}$ & $1.039063-12.42598423 .475643$ & $\mathrm{H}$ & $-1.493441-7.19772719 .229738$ \\
\hline $\mathrm{C}$ & $-0.088716-10.88039414 .603311$ & $\mathrm{~h}$ & $-0.140951-11.70417813 .891469$ \\
\hline $\mathrm{H}$ & $0.080741-9.92288314 .084086$ & $\mathrm{~h}$ & $0.206363-17.61573420 .996269$ \\
\hline $\mathrm{H}$ & $-1.101639-10.81978615 .027993$ & $\mathrm{~h}$ & $7.792373-11.78443218 .276524$ \\
\hline $\mathrm{N}$ & $2.245892-10.86846015 .655559$ & $\mathrm{~h}$ & $-0.538785-5.65666820 .835761$ \\
\hline $\mathrm{H}$ & $2.768283-10.61130314 .810488$ & & \\
\hline $\mathrm{C}$ & $0.877687-11.06860415 .734003$ & 4 & \\
\hline $\mathrm{C}$ & $2.780004-11.02885516 .881231$ & $\mathrm{ZPI}$ & at B1: 0.482805 (a.u.) \\
\hline $\mathrm{H}$ & $3.839770-10.90019817 .091662$ & Tot & QM/MM energy at B2: -3166.547607 (a.u.) \\
\hline $\mathrm{N}$ & $1.832627-11.34624117 .758447$ & $\mathrm{Fe}$ & $2.091755-11.39358119 .872281$ \\
\hline $\mathrm{C}$ & $0.643964-11.37057017 .056962$ & $\mathrm{P}$ & $-0.440248-10.60677421 .713222$ \\
\hline $\mathrm{H}$ & $-0.304691-11.58065217 .542820$ & $\mathrm{C}$ & $0.876970-10.33421222 .943980$ \\
\hline $\mathrm{C}$ & $1.205928-17.18970720 .910034$ & $\mathrm{C}$ & $1.747864-11.55525223 .213067$ \\
\hline $\mathrm{H}$ & $1.686319-17.16120421 .899987$ & $\mathrm{O}$ & $2.484092-11.91216222 .037862$ \\
\hline $\mathrm{H}$ & $1.760018-17.93434720 .314253$ & $\mathrm{C}$ & $2.678147-11.34820124 .406424$ \\
\hline $\mathrm{N}$ & $1.383141-15.67439518 .869172$ & $\mathrm{O}$ & $-1.419074-11.67288422 .211121$ \\
\hline $\mathrm{H}$ & $1.241449-16.38459818 .132138$ & $\mathrm{O}$ & $0.226288-11.12206120 .353074$ \\
\hline $\mathrm{C}$ & $1.317611-15.84837320 .242779$ & $\mathrm{O}$ & $-1.075531-9.19991521 .498793$ \\
\hline $\mathrm{C}$ & $1.619347-14.37482418 .606798$ & $\mathrm{H}$ & $0.353343-10.03181523 .867351$ \\
\hline $\mathrm{H}$ & $1.741748-13.98434817 .600045$ & $\mathrm{H}$ & $2.090794-11.13587725 .313831$ \\
\hline $\mathrm{N}$ & $1.713749-13.67907219 .730642$ & $\mathrm{H}$ & $3.285799-12.24355024 .601427$ \\
\hline $\mathrm{C}$ & $1.529978-14.58611820 .758770$ & $\mathrm{H}$ & $3.362452-10.50282624 .226984$ \\
\hline $\mathrm{H}$ & $1.608187-14.27800921 .797023$ & $\mathrm{H}$ & $1.074046-12.40565223 .427295$ \\
\hline $\mathrm{C}$ & $6.796325-11.96962618 .678590$ & $\mathrm{C}$ & $-0.093334-10.87818114 .577894$ \\
\hline $\mathrm{H}$ & $6.118994-11.56801017 .908089$ & $\mathrm{H}$ & $0.062526-9.91843814 .058102$ \\
\hline $\mathrm{H}$ & $6.666017-13.06303518 .701605$ & $\mathrm{H}$ & $-1.103259-10.82737715 .010588$ \\
\hline $\mathrm{C}$ & $6.476307-11.34016820 .027513$ & $\mathrm{~N}$ & $2.254183-10.88536415 .604377$ \\
\hline $\mathrm{H}$ & $7.153031-11.66721820 .832336$ & $\mathrm{H}$ & $2.772621-10.64677614 .751521$ \\
\hline $\mathrm{H}$ & $6.580253-10.23992119 .981209$ & $\mathrm{C}$ & $0.883357-11.05496115 .701028$ \\
\hline $\mathrm{C}$ & $5.057593-11.60030320 .522057$ & $\mathrm{C}$ & $2.797805-11.01403716 .829769$ \\
\hline $\mathrm{O}$ & $4.847674-11.46711021 .742489$ & $\mathrm{H}$ & $3.861341-10.89614817 .027152$ \\
\hline $\mathrm{O}$ & $4.153468-11.91765119 .661332$ & $\mathrm{~N}$ & $1.851351-11.28375217 .722033$ \\
\hline $\mathrm{O}$ & $2.823882-9.75561420 .046990$ & $\mathrm{C}$ & $0.655284-11.30898717 .034724$ \\
\hline $\mathrm{H}$ & $1.572224-9.53939722 .615821$ & $\mathrm{H}$ & $-0.291715-11.48527217 .535730$ \\
\hline $\mathrm{H}$ & $3.440282-11.78509222 .052987$ & $\mathrm{C}$ & $1.291966-17.02933820 .891854$ \\
\hline $\mathrm{O}$ & $1.451988-8.01209717 .610240$ & $\mathrm{H}$ & $1.778652-16.97844921 .877930$ \\
\hline $\mathrm{H}$ & $1.932035-7.79003816 .777687$ & $\mathrm{H}$ & $1.874559-17.75081220 .293863$ \\
\hline $\mathrm{H}$ & $2.076781-8.57247318 .100913$ & $\mathrm{~N}$ & $1.388291-15.51603618 .844030$ \\
\hline $\mathrm{C}$ & $0.124254-6.27242220 .228066$ & $\mathrm{H}$ & $1.252162-16.23623218 .115720$ \\
\hline $\mathrm{H}$ & $0.548109-5.65254819 .426281$ & $\mathrm{C}$ & $1.353630-15.68538020 .220036$ \\
\hline $\mathrm{H}$ & $0.946205-6.66103620 .843543$ & $\mathrm{C}$ & $1.610777-14.21618018 .572465$ \\
\hline $\mathrm{N}$ & $-0.564999-7.43992019 .615506$ & $\mathrm{H}$ & $1.711375-13.83177017 .562070$ \\
\hline $\mathrm{H}$ & $0.009280-7.80330518 .833374$ & $\mathrm{~N}$ & $1.724972-13.51496919 .692401$ \\
\hline $\mathrm{H}$ & $-0.729571-8.21006020 .350481$ & $\mathrm{C}$ & $1.567962-14.41945820 .727582$ \\
\hline
\end{tabular}


H $\quad 1.673975-14.10850121 .761748$

C $\quad 6.759920-11.93559718 .645351$

H $\quad 6.102323-11.54485217 .851551$

H $6.613993-13.02563718 .701437$

C $\quad 6.439052-11.25397519 .965928$

H $\quad 7.072340-11.60712320 .795906$

H $\quad 6.625056-10.16573219 .907094$

C $\quad 4.998820-11.38648720 .454045$

O $\quad 4.774476-10.99865621 .614731$

O $\quad 4.104177-11.86573519 .657810$

O $\quad 2.475048-9.81342519 .951620$

H $\quad 1.502847-9.48937822 .611304$

H $\quad 3.417473-11.49323122 .028133$

O $\quad 1.362476-8.12542017 .735575$

H $\quad 1.922621-7.92918516 .949092$

H $\quad 1.859465-8.77146118 .272025$

C $\quad 0.107226-6.26635920 .262523$

H $\quad 0.554586-5.64825519 .471716$

H $\quad 0.912078-6.67643120 .886842$

$\mathrm{N} \quad-0.598818-7.41172919 .631241$

H $\quad-0.028883-7.76531418 .839675$

H $\quad-0.758573-8.20310620 .345068$

$\mathrm{H} \quad-1.528050-7.15375819 .259017$

h $\quad-0.141648-11.70246913 .866359$

h $\quad 0.314780-17.50245520 .988466$

h $\quad 7.765082-11.76979318 .257763$

h $\quad-0.553001-5.63980820 .862191$

\section{TS4}

ZPE at B1: 0.477532 (a.u.)

Total QM/MM energy at B2: -3166.496944 (a.u.)

$\mathrm{Fe} \quad 2.049201-11.60465920 .006958$

$\mathrm{P} \quad-0.362886-10.59269521 .705761$

C $\quad 1.144738-10.22802022 .646764$

C $\quad 1.851213-11.41167923 .274818$

O $2.410633-12.23361022 .243370$

C $\quad 2.884707-11.02095124 .328919$

O $\quad-1.275370-11.59440922 .410291$

O $\quad 0.169914-11.23978220 .339278$

O $\quad-1.021971-9.20498421 .448854$

H $\quad 1.031500-9.36005823 .307390$

H $\quad 2.404627-10.43744825 .130459$

H $\quad 3.330950-11.91740924 .785655$

H $\quad 3.692963-10.42149923 .883122$
$1.067850-12.02576723 .762355$

C $\quad-0.050053-10.86623014 .631427$

H $\quad 0.117422-9.91007514 .108313$

H $\quad-1.060216-10.80133915 .062108$

N $\quad 2.294150-10.89670715 .676915$

H $\quad 2.829495-10.69257614 .827003$

C $\quad 0.919899-11.04542715 .760705$

C $\quad 2.817729-11.00455516 .915885$

$\mathrm{H} \quad 3.881413-10.90502417 .121435$

N $\quad 1.858816-11.23144717 .808445$

C $\quad 0.674931-11.26107417 .098623$

H $\quad-0.281218-11.41488617 .589188$

C $\quad 1.227084-17.15224220 .858055$

H $1.734191-17.12304921 .834476$

H $\quad 1.774123-17.88471320 .241246$

N $\quad 1.366696-15.60937818 .828108$

H $\quad 1.229783-16.31479318 .084889$

C $\quad 1.305298-15.80316620 .200435$

C $\quad 1.595527-14.30584618 .581663$

H $\quad 1.713754-13.89658017 .582340$

N $\quad 1.686382-13.62890819 .717984$

C $\quad 1.503302-14.54921120 .736699$

H $\quad 1.587099-14.24919021 .776658$

C $\quad 6.711186-11.99624418 .653473$

H $\quad 6.045500-11.61546117 .862147$

H $\quad 6.601105-13.09135618 .688048$

C $\quad 6.358454-11.35460719 .987959$

H $\quad 7.052586-11.63118920 .796592$

H $\quad 6.408621-10.25195819 .913603$

C $\quad 4.959930-11.67046120 .511437$

O $4.795246-11.59501421 .739659$

O $4.032451-11.97608719 .666660$

O $\quad 2.475737-9.97393220 .515207$

H $\quad 1.924423-9.86512421 .580728$

H $3.403206-12.01028222 .142315$

O $\quad 1.340631-8.12465117 .689992$

H $\quad 1.897907-7.91444116 .902726$

H $\quad 1.774203-8.88480818 .112707$

C $\quad 0.115495-6.24834220 .242984$

H $\quad 0.561198-5.62342719 .456756$

H $\quad 0.922182-6.66257620 .862121$

N $\quad-0.592565-7.38900519 .606147$

$\mathrm{H} \quad-0.033090-7.73027818 .802598$

H $\quad-0.733548-8.19230420 .311202$ 
H $\quad-1.530048-7.13352619 .253357$

h $\quad-0.111924-11.69012513 .920486$

h $\quad 0.236522-17.59278020 .971101$

h $\quad 7.713184-11.79823518 .272868$

h $\quad-0.544454-5.62642920 .847762$

\section{5}

ZPE at B1: 0.486444 (a.u.)

Total QM/MM energy at B2: -3166.529068 (a.u.)

$\mathrm{Fe} \quad 2.099261-11.54788819 .842648$

P $\quad-0.443445-10.66151021 .709736$

C $\quad 0.772284-10.52706023 .018569$

C $\quad 1.803209-11.57378523 .287657$

O $2.491744-11.93747022 .088155$

C $\quad 2.769220-11.18040324 .408013$

O $\quad-1.477998-11.72004622 .117307$

O $\quad 0.265076-11.11391620 .360538$

O $\quad-1.029287-9.22796721 .547418$

H $\quad 0.498953-9.85331423 .839640$

H $\quad 2.205750-10.92054225 .317974$

H $\quad 3.446082-12.01040824 .654776$

H $\quad 3.380032-10.31285624 .111380$

H $\quad 1.253399-12.48470923 .619708$

C $\quad-0.085212-10.88370314 .583906$

H $\quad 0.076807-9.92159814 .070626$

H $\quad-1.093153-10.82745815 .020574$

N $\quad 2.264243-10.90153015 .605409$

H $\quad 2.779860-10.64048714 .757715$

C $\quad 0.895197-11.08065715 .701247$

C $\quad 2.810590-11.05811216 .827545$

H $\quad 3.874983-10.93977517 .020358$

$\mathrm{N} \quad 1.870532-11.35749417 .715278$

C $\quad 0.673758-11.37051517 .028848$

H $\quad-0.272609-11.56183717 .526399$

C $\quad 1.256026-17.06133020 .916572$

H $\quad 1.736252-17.01534221 .905946$

H $\quad 1.836698-17.78664920 .321828$

$\mathrm{N} \quad 1.384348-15.55073018 .868304$

H $\quad 1.245724-16.26967218 .138395$

C $\quad 1.332544-15.71745120 .245737$

C $\quad 1.620921-14.25712818 .593097$

H $\quad 1.735681-13.87191717 .584753$

N $\quad 1.725578-13.55567919 .716262$

C $\quad 1.549557-14.45361820 .755310$
$1.647289-14.13774521 .788922$

C $\quad 6.768700-11.96517118 .645128$

H $\quad 6.107087-11.58688217 .848865$

H $\quad 6.633222-13.05602218 .707517$

C $\quad 6.438527-11.27865419 .960518$

H $\quad 7.090240-11.59999620 .789340$

H $\quad 6.589295-10.18624619 .884764$

C $\quad 5.006995-11.45381320 .458075$

O $4.778190-11.05573421 .616834$

O $\quad 4.126696-11.97588319 .677053$

O $\quad 2.573627-9.76250219 .875357$

$\mathrm{H} \quad 2.391958-9.43706720 .771527$

H $\quad 3.442333-11.56084222 .056520$

O $\quad 1.371135-8.21329417 .879215$

H $\quad 1.935868-8.01127417 .100379$

H $\quad 1.882213-8.80896618 .477656$

C $\quad 0.101739-6.27896920 .279840$

H $\quad 0.559034-5.67114819 .486702$

H $\quad 0.900586-6.67865820 .919166$

N $\quad-0.592939-7.43434819 .656948$

H $\quad-0.002774-7.79719718 .876955$

H $\quad-0.749350-8.21734820 .374330$

H $\quad-1.519826-7.18695019 .272316$

h $\quad-0.143139-11.70221213 .866442$

h $\quad 0.274102-17.52617221 .004967$

h $\quad 7.771695-11.78981918 .256131$

h $\quad-0.560547-5.64437020 .868676$

TS5

ZPE at B1: 0.480858 (a.u.)

Total QM/MM energy at B2: -3166.505547 (a.u.)

Fe $\quad 2.020736-11.52367719 .892805$

P $\quad-0.410019-10.55068921 .649553$

C $\quad 1.134880-10.16227222 .542079$

C $\quad 1.877629-11.35461323 .114027$

O $\quad 2.369922-12.16082522 .036575$

C $\quad 2.973415-10.96982324 .104265$

O $\quad-1.260491-11.56957922 .403773$

O $\quad 0.083950-11.15415520 .258491$

O $\quad-1.096112-9.16736921 .446649$

H $\quad 0.977833-9.36138523 .276181$

H $\quad 2.540423-10.38198224 .928950$

H $\quad 3.439125-11.86609324 .540186$

H $\quad 3.758250-10.37734523 .611368$ 


\begin{tabular}{|c|c|c|c|}
\hline $\mathrm{H}$ & $1.121421-11.96617623 .644806$ & $\mathrm{H}$ & $-1.550618-7.09980319 .233177$ \\
\hline $\mathrm{C}$ & $-0.104343-10.89174014 .602386$ & $\mathrm{~h}$ & $-0.149169-11.71669913 .891402$ \\
\hline $\mathrm{H}$ & $0.055246-9.93323714 .081439$ & $\mathrm{~h}$ & $0.186781-17.64609820 .987666$ \\
\hline $\mathrm{H}$ & $-1.117443-10.83939015 .027340$ & $\mathrm{~h}$ & $7.785540-11.79292418 .237571$ \\
\hline $\mathrm{N}$ & $2.237230-10.90557315 .645892$ & $\mathrm{~h}$ & $-0.556928-5.61819720 .850943$ \\
\hline $\mathrm{H}$ & $2.761779-10.67450914 .794683$ & & \\
\hline $\mathrm{C}$ & $0.862889-11.06527315 .734475$ & 6 & \\
\hline $\mathrm{C}$ & $2.772409-11.03313116 .873135$ & $\mathrm{ZPI}$ & at B1: 0.484958 (a.u.) \\
\hline $\mathrm{H}$ & $3.835607-10.92900317 .080413$ & Tot & QM/MM energy at B2: -3166.549300 (a.u.) \\
\hline $\mathrm{N}$ & $1.817267-11.28779317 .764760$ & $\mathrm{Fe}$ & $2.102878-11.37126119 .845044$ \\
\hline $\mathrm{C}$ & $0.624509-11.30718217 .067488$ & $\mathrm{P}$ & $-0.512725-10.63447121 .687458$ \\
\hline $\mathrm{H}$ & $-0.324942-11.47219317 .567404$ & $\mathrm{C}$ & $0.667521-10.58665823 .028322$ \\
\hline $\mathrm{C}$ & $1.188362-17.22754920 .889081$ & $\mathrm{C}$ & $1.837592-11.49831523 .185769$ \\
\hline $\mathrm{H}$ & $1.681396-17.20112821 .872818$ & $\mathrm{O}$ & $2.485888-11.75247821 .936514$ \\
\hline $\mathrm{H}$ & $1.730235-17.97425420 .285176$ & $\mathrm{C}$ & $2.824606-10.98678324 .239880$ \\
\hline $\mathrm{N}$ & $1.376415-15.71042918 .849906$ & $\mathrm{O}$ & $-1.557365-11.71717421 .983021$ \\
\hline $\mathrm{H}$ & $1.242763-16.41969618 .110677$ & $\mathrm{O}$ & $0.252050-10.98728320 .336777$ \\
\hline $\mathrm{C}$ & $1.292705-15.88575920 .221963$ & $\mathrm{O}$ & $-1.084408-9.19188721 .578885$ \\
\hline $\mathrm{C}$ & $1.598911-14.40708018 .592259$ & $\mathrm{H}$ & $0.313527-10.07632823 .932723$ \\
\hline $\mathrm{H}$ & $1.731422-14.01460417 .587110$ & $\mathrm{H}$ & $2.304010-10.84113425 .199233$ \\
\hline $\mathrm{N}$ & $1.667916-13.71203819 .718008$ & $\mathrm{H}$ & $3.638012-11.70682624 .400822$ \\
\hline $\mathrm{C}$ & $1.477311-14.62189020 .742827$ & $\mathrm{H}$ & $3.267497-10.02820023 .927321$ \\
\hline $\mathrm{H}$ & $1.531238-14.30927421 .781161$ & $\mathrm{H}$ & $1.440484-12.47607523 .548609$ \\
\hline $\mathrm{C}$ & $6.778948-11.99423518 .604058$ & $\mathrm{C}$ & $-0.121304-10.89258414 .575953$ \\
\hline $\mathrm{H}$ & $6.122634-11.59896417 .812216$ & $\mathrm{H}$ & $0.027756-9.93061014 .058491$ \\
\hline $\mathrm{H}$ & $6.668301-13.08998218 .615966$ & $\mathrm{H}$ & $-1.131120-10.84893515 .009590$ \\
\hline $\mathrm{C}$ & $6.402494-11.37939319 .944767$ & $\mathrm{~N}$ & $2.226808-10.87375315 .597788$ \\
\hline $\mathrm{H}$ & $7.079363-11.67191920 .761880$ & $\mathrm{H}$ & $2.736649-10.60941314 .746548$ \\
\hline $\mathrm{H}$ & $6.446868-10.27534119 .890213$ & $\mathrm{C}$ & $0.858472-11.06737915 .696689$ \\
\hline $\mathrm{C}$ & $4.991861-11.72385920 .410418$ & $\mathrm{C}$ & $2.777190-11.01357316 .816855$ \\
\hline $\mathrm{O}$ & $4.804482-11.78169221 .640416$ & $\mathrm{H}$ & $3.839461-10.87867217 .012099$ \\
\hline $\mathrm{O}$ & $4.074499-11.91470319 .528957$ & $\mathrm{~N}$ & $1.839345-11.31434817 .709906$ \\
\hline $\mathrm{O}$ & $2.503579-9.79468920 .466129$ & $\mathrm{C}$ & $0.640565-11.34673217 .026021$ \\
\hline $\mathrm{H}$ & $1.846420-9.71247221 .624595$ & $\mathrm{H}$ & $-0.302292-11.54644817 .527182$ \\
\hline $\mathrm{H}$ & $3.398371-12.05820721 .964233$ & $\mathrm{C}$ & $1.272654-17.10552620 .910095$ \\
\hline $\mathrm{O}$ & $1.389981-8.12336317 .802609$ & $\mathrm{H}$ & $1.753194-17.06552621 .899673$ \\
\hline $\mathrm{H}$ & $1.968688-7.95689617 .022023$ & $\mathrm{H}$ & $1.841958-17.84054120 .316403$ \\
\hline $\mathrm{H}$ & $1.883156-8.72153518 .390096$ & $\mathrm{~N}$ & $1.418419-15.60002418 .862444$ \\
\hline $\mathrm{C}$ & $0.097410-6.23954120 .239519$ & $\mathrm{H}$ & $1.280054-16.31803618 .132510$ \\
\hline $\mathrm{H}$ & $0.543398-5.61409719 .453970$ & $\mathrm{C}$ & $1.363507-15.76415920 .237986$ \\
\hline $\mathrm{H}$ & $0.904865-6.66372520 .851001$ & $\mathrm{C}$ & $1.648716-14.30082218 .590194$ \\
\hline $\mathrm{N}$ & $-0.624175-7.37015419 .601894$ & $\mathrm{H}$ & $1.763390-13.91910217 .579396$ \\
\hline $\mathrm{H}$ & $-0.057546-7.73156118 .812803$ & $\mathrm{~N}$ & $1.747983-13.59499119 .707206$ \\
\hline $\mathrm{H}$ & $-0.792351-8.16338520 .313048$ & $\mathrm{C}$ & $1.575028-14.49512820 .742053$ \\
\hline
\end{tabular}


H $\quad 1.652569-14.18328021 .779958$

C $\quad 6.827657-11.94087618 .624407$

H $\quad 6.165524-11.55329817 .833225$

H $\quad 6.689451-13.03231218 .675703$

C $\quad 6.498018-11.26778819 .947612$

H $\quad 7.154192-11.58782420 .772417$

H $\quad 6.630814-10.17228019 .875018$

C $\quad 5.069461-11.47241020 .433168$

O $4.862726-11.24458721 .646489$

O $\quad 4.161624-11.83657119 .604846$

O $\quad 2.654731-9.51276419 .865837$

H $\quad 2.205011-9.06619720 .598836$

H $\quad 3.518644-11.53466021 .946241$

O $\quad 1.366541-8.14096417 .845645$

H $\quad 1.907858-7.92825917 .053113$

H $\quad 1.938677-8.66849518 .459585$

C $\quad 0.082003-6.27095420 .270187$

H $\quad 0.527060-5.66002919 .472867$

H $\quad 0.890731-6.66799620 .898641$

$\mathrm{N} \quad-0.616518-7.42665519 .651042$

H $\quad-0.028085-7.79031818 .869823$

H $\quad-0.783237-8.19963920 .373665$

H $\quad-1.542523-7.17566319 .265221$

h $\quad-0.165600-11.71460213 .861537$

h $\quad 0.283346-17.55444820 .998443$

h $\quad 7.831439-11.76909718 .235843$

h $\quad-0.575186-5.64265020 .871356$

\section{TS6}

ZPE at B1: 0.485138 (a.u.)

Total QM/MM energy at B2: -3166.516428 (a.u.)

$\mathrm{Fe} \quad 1.906877-11.39049719 .930527$

P $\quad-0.573961-10.54026421 .724209$

C $\quad 0.778735-10.34563222 .931187$

C $\quad 1.772207-11.51134222 .966787$

O $\quad 2.430316-11.64181221 .736870$

C $\quad 2.783897-11.33893224 .102062$

O $\quad-1.500413-11.68474922 .137887$

O $\quad 0.083622-10.87798420 .299335$

O $\quad-1.239814-9.13975321 .606815$

H $\quad 0.293414-10.21100223 .913285$

H $\quad 2.276618-11.26270525 .077642$

H $\quad 3.469528-12.19624924 .141026$

H $\quad 3.387292-10.42927623 .945788$
H $\quad 1.181402-12.43340423 .170936$

C $\quad-0.213760-10.89890714 .539174$

H $\quad-0.072655-9.93604114 .021433$

H $\quad-1.234888-10.87520014 .946953$

N $\quad 2.106984-10.83708915 .601484$

H $\quad 2.619251-10.55638514 .756351$

C $\quad 0.743555-11.05523515 .681409$

C $\quad 2.642927-10.98670416 .828729$

H $\quad 3.702090-10.84339617 .035663$

N $\quad 1.704032-11.31700917 .706806$

C $\quad 0.516299-11.35752317 .005952$

H $\quad-0.430473-11.57450217 .494157$

C $\quad 1.300229-17.08524720 .888760$

H $\quad 1.786806-17.03966921 .875081$

H $\quad 1.869925-17.81771020 .292298$

N $\quad 1.417534-15.58057818 .837034$

$\mathrm{H} \quad 1.282571-16.30212318 .109468$

C $\quad 1.375520-15.74454620 .213724$

C $\quad 1.620861-14.27906018 .559551$

H $\quad 1.716467-13.89261017 .548818$

N $\quad 1.717005-13.57167319 .676675$

C $\quad 1.571900-14.47350420 .715627$

H $\quad 1.653360-14.15661821 .751656$

C $\quad 6.969503-11.87409718 .573598$

H $\quad 6.343438-11.47293917 .759520$

H $\quad 6.778988-12.95519318 .643689$

C $\quad 6.635418-11.14564719 .866486$

H $\quad 7.100348-11.60827420 .754744$

H $\quad 6.994078-10.10230719 .863663$

C $\quad 5.148667-11.07377420 .120945$

O $\quad 4.844257-10.25047721 .097526$

O $\quad 4.306859-11.69173019 .466039$

O $\quad 2.596511-9.53972919 .786472$

H $\quad 1.318044-9.41244622 .692603$

H $\quad 3.857389-10.16739221 .130623$

O $\quad 1.309564-8.10834417 .795542$

H $\quad 1.892597-7.91137217 .029670$

H $\quad 1.849166-8.64507118 .427907$

C $\quad 0.057119-6.27938220 .265529$

H $\quad 0.490778-5.67154619 .459585$

H $\quad 0.875494-6.68877820 .872849$

N $\quad-0.669848-7.42399019 .657824$

$\mathrm{H} \quad-0.100136-7.80401418 .874917$

H $\quad-0.863003-8.18272620 .390433$ 


\begin{tabular}{|c|c|c|c|}
\hline $\mathrm{H}$ & $-1.591761-7.15206519 .275837$ & $\mathrm{H}$ & $1.634230-14.26159821 .778210$ \\
\hline $\mathrm{h}$ & $-0.218967-11.72619013 .829497$ & $\mathrm{C}$ & $6.893878-11.86357118 .644398$ \\
\hline $\mathrm{h}$ & $0.313993-17.53913920 .985659$ & $\mathrm{H}$ & $6.275593-11.48216617 .814845$ \\
\hline $\mathrm{h}$ & $7.985044-11.74121418 .200657$ & $\mathrm{H}$ & $6.703631-12.94288818 .737033$ \\
\hline $\mathrm{h}$ & $-0.588051-5.64822920 .876649$ & $\mathrm{C}$ & $6.542355-11.10049719 .919561$ \\
\hline & & $\mathrm{H}$ & $6.899553-11.61649420 .828366$ \\
\hline 7 & & $\mathrm{H}$ & $6.993185-10.09594119 .948887$ \\
\hline & at B1: 0.483823 (a.u.) & $\mathrm{C}$ & $5.047515-10.90170620 .062608$ \\
\hline & QM/MM energy at B2: -3166.518100 (a.u.) & $\mathrm{O}$ & $4.712782-9.85916920 .762834$ \\
\hline $\mathrm{Fe}$ & $1.921116-11.51403919 .990146$ & $\mathrm{O}$ & $4.221548-11.65844819 .523072$ \\
\hline $\mathrm{P}$ & $-0.567580-10.59253821 .728885$ & $\mathrm{O}$ & $2.443143-9.53093719 .912140$ \\
\hline $\mathrm{C}$ & $0.796325-10.36546122 .919027$ & $\mathrm{H}$ & $1.351845-9.45723722 .626446$ \\
\hline $\mathrm{C}$ & $1.768656-11.54452723 .019957$ & $\mathrm{H}$ & $3.680258-9.67543220 .595474$ \\
\hline $\mathrm{O}$ & $2.371235-11.80507721 .782536$ & $\mathrm{O}$ & $1.225999-8.13537717 .820807$ \\
\hline $\mathrm{C}$ & $2.833125-11.29048124 .090278$ & $\mathrm{H}$ & $1.870412-7.95833317 .099471$ \\
\hline $\mathrm{O}$ & $-1.497402-11.72085322 .179032$ & $\mathrm{H}$ & $1.688008-8.69365218 .487779$ \\
\hline $\mathrm{O}$ & $0.077324-10.96993020 .311913$ & $\mathrm{C}$ & $0.027303-6.29483920 .294506$ \\
\hline $\mathrm{O}$ & $-1.233572-9.19139421 .597916$ & $\mathrm{H}$ & $0.471573-5.69434219 .488620$ \\
\hline $\mathrm{H}$ & $0.319121-10.16633623 .894229$ & $\mathrm{H}$ & $0.837743-6.71528820 .904791$ \\
\hline $\mathrm{H}$ & $2.370028-11.06076725 .063778$ & $\mathrm{~N}$ & $-0.717101-7.42861119 .687252$ \\
\hline $\mathrm{H}$ & $3.474051-12.17314224 .220873$ & $\mathrm{H}$ & $-0.168494-7.79469218 .884688$ \\
\hline $\mathrm{H}$ & $3.473954-10.44544623 .789495$ & $\mathrm{H}$ & $-0.890058-8.20438520 .409619$ \\
\hline $\mathrm{H}$ & $1.170937-12.43336323 .324784$ & $\mathrm{H}$ & $-1.648755-7.14955419 .332962$ \\
\hline $\mathrm{C}$ & $-0.185987-10.89609914 .600505$ & $\mathrm{~h}$ & $-0.203818-11.71633113 .882892$ \\
\hline $\mathrm{H}$ & $-0.033853-9.93005814 .091751$ & $\mathrm{~h}$ & $0.239431-17.61190920 .992948$ \\
\hline $\mathrm{H}$ & $-1.205639-10.86391315 .011426$ & $\mathrm{~h}$ & $7.908358-11.73540318 .266947$ \\
\hline $\mathrm{N}$ & $2.139495-10.88566715 .654944$ & $\mathrm{~h}$ & $-0.611873-5.65055020 .898172$ \\
\hline $\mathrm{H}$ & $2.655097-10.61062214 .810484$ & & \\
\hline $\mathrm{C}$ & $0.772106-11.07583315 .738683$ & TS7 & \\
\hline $\mathrm{C}$ & $2.675218-11.05031216 .880326$ & $\mathrm{ZPE}$ & at B1: 0.481375 (a.u.) \\
\hline $\mathrm{H}$ & $3.738994-10.94552317 .081117$ & Tota & QM/MM energy at B2: -3166.517544 (a.u.) \\
\hline $\mathrm{N}$ & $1.732341-11.36011917 .762383$ & $\mathrm{Fe}$ & $1.963443-11.56543620 .003220$ \\
\hline $\mathrm{C}$ & $0.542430-11.37509717 .063456$ & $\mathrm{P}$ & $-0.540314-10.60464221 .722287$ \\
\hline $\mathrm{H}$ & $-0.406824-11.57156917 .554781$ & $\mathrm{C}$ & $0.814436-10.37272122 .922581$ \\
\hline $\mathrm{C}$ & $1.236006-17.18032820 .899983$ & $\mathrm{C}$ & $1.781915-11.55395123 .036515$ \\
\hline $\mathrm{H}$ & $1.721999-17.14733921 .886973$ & $\mathrm{O}$ & $2.385102-11.82720121 .802548$ \\
\hline $\mathrm{H}$ & $1.790230-17.92269920 .301387$ & $\mathrm{C}$ & $2.845870-11.29713824 .106440$ \\
\hline $\mathrm{N}$ & $1.381547-15.66637618 .855033$ & $\mathrm{O}$ & $-1.478217-11.72594122 .174617$ \\
\hline $\mathrm{H}$ & $1.237405-16.37904618 .120501$ & $\mathrm{O}$ & $0.114481-10.99075220 .314137$ \\
\hline $\mathrm{C}$ & $1.336330-15.83896120 .230758$ & $\mathrm{O}$ & $-1.203093-9.20165021 .582947$ \\
\hline $\mathrm{C}$ & $1.602210-14.36656618 .585227$ & $\mathrm{H}$ & $0.332963-10.16661823 .894036$ \\
\hline $\mathrm{H}$ & $1.700684-13.97439717 .576755$ & $\mathrm{H}$ & $2.382752-11.04941925 .075352$ \\
\hline $\mathrm{N}$ & $1.708134-13.66957719 .707636$ & $\mathrm{H}$ & $3.476278-12.18542224 .249775$ \\
\hline $\mathrm{C}$ & $1.548667-14.57469720 .741420$ & $\mathrm{H}$ & $3.495172-10.46280823 .794350$ \\
\hline
\end{tabular}




\begin{tabular}{|c|c|c|c|}
\hline $\mathrm{H}$ & $1.180207-12.43868823 .345245$ & $\mathrm{H}$ & $-1.637881-7.16860619 .328905$ \\
\hline $\mathrm{C}$ & $-0.170029-10.89047914 .625846$ & $\mathrm{~h}$ & $-0.190637-11.70876213 .906086$ \\
\hline $\mathrm{H}$ & $-0.019404-9.92390714 .117606$ & $\mathrm{~h}$ & $0.226847-17.63463120 .994716$ \\
\hline $\mathrm{H}$ & $-1.188805-10.85864715 .039067$ & $\mathrm{~h}$ & $7.861759-11.72229218 .285346$ \\
\hline $\mathrm{N}$ & $2.156531-10.86324315 .676994$ & $\mathrm{~h}$ & $-0.604907-5.65558920 .885110$ \\
\hline $\mathrm{H}$ & $2.668489-10.58393814 .831759$ & & \\
\hline $\mathrm{C}$ & $0.791254-11.06847515 .761711$ & 8 & \\
\hline $\mathrm{C}$ & $2.695962-11.02833216 .900546$ & $\mathrm{ZPI}$ & at B1: 0.483237 (a.u.) \\
\hline $\mathrm{H}$ & $3.758865-10.91444317 .101129$ & Tot & QM/MM energy at B2: -3166.518785 (a.u.) \\
\hline $\mathrm{N}$ & $1.757145-11.35355217 .782896$ & $\mathrm{Fe}$ & $2.011884-11.63185820 .020657$ \\
\hline $\mathrm{C}$ & $0.566723-11.37665217 .085550$ & $\mathrm{P}$ & $-0.524081-10.60370421 .713737$ \\
\hline $\mathrm{H}$ & $-0.379236-11.58837317 .576474$ & $\mathrm{C}$ & $0.803957-10.39195522 .944917$ \\
\hline $\mathrm{C}$ & $1.227322-17.21169420 .903937$ & $\mathrm{C}$ & $1.760272-11.58176323 .065879$ \\
\hline $\mathrm{H}$ & $1.712518-17.18502621 .891493$ & $\mathrm{O}$ & $2.363526-11.86885621 .835087$ \\
\hline $\mathrm{H}$ & $1.776493-17.95689620 .304385$ & $\mathrm{C}$ & $2.822741-11.32991824 .137963$ \\
\hline $\mathrm{N}$ & $1.384408-15.69433618 .862486$ & $\mathrm{O}$ & $-1.469995-11.73387322 .130846$ \\
\hline $\mathrm{H}$ & $1.238606-16.40489518 .126002$ & $\mathrm{O}$ & $0.159889-10.96973120 .313714$ \\
\hline $\mathrm{C}$ & $1.336679-15.86986420 .237691$ & $\mathrm{O}$ & $-1.187794-9.20139621 .579836$ \\
\hline $\mathrm{C}$ & $1.609259-14.39526218 .594801$ & $\mathrm{H}$ & $0.305276-10.19404323 .909200$ \\
\hline $\mathrm{H}$ & $1.710354-14.00057717 .587301$ & $\mathrm{H}$ & $2.356406-11.06571725 .100921$ \\
\hline $\mathrm{N}$ & $1.715320-13.70151419 .719384$ & $\mathrm{H}$ & $3.438795-12.22575124 .296033$ \\
\hline $\mathrm{C}$ & $1.551926-14.60782420 .751583$ & $\mathrm{H}$ & $3.486112-10.50914323 .820313$ \\
\hline $\mathrm{H}$ & $1.636603-14.29765521 .789388$ & $\mathrm{H}$ & $1.148467-12.45940023 .374299$ \\
\hline $\mathrm{C}$ & $6.846174-11.84625018 .661228$ & $\mathrm{C}$ & $-0.167301-10.90739414 .652931$ \\
\hline $\mathrm{H}$ & $6.232837-11.46224617 .828960$ & $\mathrm{H}$ & $-0.010950-9.93901214 .149994$ \\
\hline $\mathrm{H}$ & $6.651520-12.92518718 .750620$ & $\mathrm{H}$ & $-1.185486-10.87158415 .067377$ \\
\hline $\mathrm{C}$ & $6.488514-11.08658619 .939042$ & $\mathrm{~N}$ & $2.159386-10.88759415 .704911$ \\
\hline $\mathrm{H}$ & $6.816920-11.62687120 .844466$ & $\mathrm{H}$ & $2.672160-10.60395814 .861907$ \\
\hline $\mathrm{H}$ & $6.965216-10.09527419 .988713$ & $\mathrm{C}$ & $0.794370-11.09729115 .786930$ \\
\hline $\mathrm{C}$ & $4.993146-10.84619220 .067561$ & $\mathrm{C}$ & $2.698125-11.06130416 .927505$ \\
\hline $\mathrm{O}$ & $4.657298-9.78429920 .700495$ & $\mathrm{H}$ & $3.760355-10.94615517 .130898$ \\
\hline $\mathrm{O}$ & $4.167488-11.64052319 .549418$ & $\mathrm{~N}$ & $1.759027-11.39380717 .807611$ \\
\hline $\mathrm{O}$ & $2.424707-9.49981819 .932585$ & $\mathrm{C}$ & $0.569541-11.41686117 .108063$ \\
\hline $\mathrm{H}$ & $1.375476-9.46785322 .629122$ & $\mathrm{H}$ & $-0.376473-11.63380217 .596337$ \\
\hline $\mathrm{H}$ & $3.495894-9.57338020 .487903$ & $\mathrm{C}$ & $1.194138-17.25668520 .918422$ \\
\hline $\mathrm{O}$ & $1.265855-8.07731317 .763366$ & $\mathrm{H}$ & $1.673811-17.23654921 .908824$ \\
\hline $\mathrm{H}$ & $1.891751-7.90613517 .023243$ & $\mathrm{H}$ & $1.738441-18.00681420 .320838$ \\
\hline $\mathrm{H}$ & $1.764317-8.59567118 .428954$ & $\mathrm{~N}$ & $1.381607-15.73605418 .881519$ \\
\hline $\mathrm{C}$ & $0.036018-6.29564820 .278806$ & $\mathrm{H}$ & $1.236342-16.44293118 .141239$ \\
\hline $\mathrm{H}$ & $0.472679-5.69499619 .469064$ & $\mathrm{C}$ & $1.320020-15.91494920 .255821$ \\
\hline $\mathrm{H}$ & $0.852226-6.70756920 .887098$ & $\mathrm{C}$ & $1.619069-14.43879818 .618692$ \\
\hline $\mathrm{N}$ & $-0.700955-7.43829119 .677365$ & $\mathrm{H}$ & $1.734858-14.04231317 .613445$ \\
\hline $\mathrm{H}$ & $-0.158972-7.79993518 .870807$ & $\mathrm{~N}$ & $1.719245-13.74910319 .746893$ \\
\hline $\mathrm{H}$ & $-0.861544-8.21816820 .401354$ & $\mathrm{C}$ & $1.538690-14.65631520 .775479$ \\
\hline
\end{tabular}


H $\quad 1.614167-14.34906821 .814894$

C $\quad 6.793318-11.81646518 .683413$

H $\quad 6.182537-11.42756717 .851416$

H $\quad 6.591730-12.89424018 .774876$

C $\quad 6.442792-11.05574619 .962043$

H $\quad 6.790012-11.58952920 .864261$

H $\quad 6.914110-10.06122020 .000909$

C $\quad 4.942548-10.81891120 .125611$

O $4.608545-9.79012420 .776711$

O $\quad 4.125693-11.63940719 .598799$

O $\quad 2.320000-9.50249520 .038254$

H $\quad 1.374690-9.48691222 .672680$

H $\quad 3.169448-9.53430220 .642940$

O $\quad 1.253227-8.11947617 .722761$

H $\quad 1.885805-7.92826316 .992042$

H $\quad 1.735042-8.68754718 .352324$

C $\quad 0.051215-6.29311320 .273062$

H $\quad 0.482997-5.68872519 .463365$

H $\quad 0.870820-6.70379520 .877043$

$\mathrm{N} \quad-0.682630-7.43824919 .672418$

H $\quad-0.138262-7.80142218 .868860$

H $\quad-0.844194-8.21612220 .397851$

H $\quad-1.618498-7.17027919 .320554$

h $\quad-0.193587-11.72141313 .928535$

h $\quad 0.187981-17.66744221 .002045$

h $\quad 7.809550-11.70062218 .306691$

h $\quad-0.591842-5.65608120 .880297$

\section{TS8}

ZPE at B1: 0.480985 (a.u.)

Total QM/MM energy at B2: -3166.509508 (a.u.)

$\mathrm{Fe} \quad 1.992303-11.61884320 .061197$

P $\quad-0.528694-10.62404021 .656432$

C $\quad 0.938794-10.25071622 .679441$

C $\quad 1.849110-11.41217923 .029906$

O $2.296610-12.04788521 .876485$

C $\quad 3.012814-11.00486123 .939177$

O $\quad-1.408698-11.69551122 .297015$

O $\quad 0.053350-11.13090420 .265187$

O $\quad-1.210585-9.23564421 .481279$

$\mathrm{H} \quad 0.663604-9.62682923 .543369$

H $\quad 2.649653-10.46005124 .825791$

H $\quad 3.549532-11.89808724 .288758$

H $\quad 3.718492-10.37039123 .380188$
$1.204044-12.12362123 .601471$

C $\quad-0.119851-10.88532114 .693636$

H $\quad 0.031957-9.92097614 .181062$

$\mathrm{H} \quad-1.135639-10.84680415 .113844$

N $\quad 2.212837-10.85872915 .744587$

$\mathrm{H}$

C $\quad 0.844548-11.05472215 .828802$

C $\quad 2.745259-10.98210416 .975784$

$\mathrm{H} \quad 3.806515-10.86080917 .181915$

N $\quad 1.798253-11.26664817 .866211$

C $\quad 0.611359-11.31366417 .160855$

H $\quad-0.334850-11.50740617 .656522$

C $\quad 1.209705-17.25150620 .885419$

H $\quad 1.703636-17.22945721 .868679$

H $\quad 1.748075-17.99970320 .279915$

N $\quad 1.369997-15.72378118 .847719$

H $\quad 1.218699-16.42865518 .106891$

C $\quad 1.323451-15.90840320 .222407$

C $\quad 1.609885-14.42527018 .589067$

H $\quad 1.714491-14.02478017 .583801$

$\mathrm{N} \quad 1.725613-13.74224819 .719011$

C $\quad 1.551367-14.65342920 .745236$

H $\quad 1.642913-14.34129821 .781641$

C $\quad 6.770923-11.84655718 .684710$

H $\quad 6.154751-11.44058117 .864768$

H $\quad 6.571362-12.92648318 .754611$

C $\quad 6.441867-11.11091119 .981939$

H $\quad 6.785177-11.67383520 .867756$

H $\quad 6.940454-10.13071420 .044218$

C $\quad 4.956187-10.82321220 .188413$

O $4.682817-9.82285220 .897096$

O $\quad 4.088981-11.58309419 .635969$

O $\quad 2.229259-9.49562420 .550248$

H $\quad 1.539899-9.60114721 .889746$

H $\quad 3.218279-9.53097720 .841519$

O $\quad 1.331297-8.07953317 .744472$

H $\quad 1.946541-7.89448716 .995778$

$\mathrm{H} \quad 1.807710-8.69980118 .320053$

C $\quad 0.067455-6.28328420 .265490$

$\mathrm{H} \quad 0.511946-5.67552919 .465248$

H $\quad 0.878064-6.70462220 .874052$

N $\quad-0.665634-7.41924419 .646134$

$\mathrm{H} \quad-0.115169-7.77790518 .846531$

H $\quad-0.836080-8.21058620 .355008$ 
H $\quad-1.597475-7.14703819 .288466$

h $\quad-0.154456-11.70263413 .973311$

h $\quad 0.206910-17.66694020 .984833$

h $\quad 7.785944-11.72407518 .306826$

h $\quad-0.580463-5.64585320 .867113$

\section{9}

ZPE at B1: 0.483451 (a.u.)

Total QM/MM energy at B2: -3166.548672 (a.u.)

$\mathrm{Fe} \quad 2.074259-11.61084320 .021774$

P $\quad-0.534860-10.77358021 .719799$

C $\quad 0.633115-10.85945323 .069737$

C $\quad 2.003390-11.46559823 .079386$

O $2.485381-11.82442921 .827676$

C $\quad 3.002636-10.53903423 .805603$

O $\quad-1.677285-11.76530921 .966473$

O $\quad 0.188262-11.14140220 .353666$

O $\quad-0.973153-9.27368721 .645584$

$\mathrm{H} \quad 0.244773-10.51309524 .036444$

H $\quad 2.660269-10.31669424 .829466$

H $\quad 3.990829-11.01605823 .854894$

H $\quad 3.116800-9.59465023 .251240$

H $\quad 1.931729-12.39203523 .703519$

C $\quad-0.156943-10.91944914 .678659$

$\mathrm{H} \quad-0.012287-9.94887514 .175723$

H $\quad-1.173143-10.89221615 .099210$

$\mathrm{N} \quad 2.172515-10.85466815 .719935$

H $\quad 2.676551-10.56748814 .872639$

C $\quad 0.812994-11.09630315 .807522$

C $\quad 2.718865-11.00586216 .941880$

H $\quad 3.777484-10.85700217 .143948$

N $\quad 1.790899-11.35503217 .827359$

C $\quad 0.600369-11.41280817 .130997$

H $\quad-0.337751-11.65401817 .623118$

C $\quad 1.236760-17.25317020 .900156$

H $\quad 1.728807-17.24819121 .884894$

H $\quad 1.767462-18.00245520 .288754$

N $\quad 1.416703-15.71872218 .874505$

H $\quad 1.256907-16.42074618 .132613$

C $\quad 1.370337-15.90640120 .248804$

C $\quad 1.676993-14.42348718 .618989$

H $\quad 1.788741-14.02144617 .615361$

N $\quad 1.805040-13.74420019 .750493$

C $\quad 1.618508-14.65477820 .774011$
$1.705597-14.35504521 .815337$

C $\quad 6.812774-11.82470318 .672968$

$\mathrm{H}$

$6.200342-11.43698317 .841272$

H $\quad 6.610156-12.90276418 .763153$

C $\quad 6.470106-11.06089719 .952024$

H $\quad 6.782511-11.61685020 .854148$

H $\quad 6.978932-10.08581420 .011067$

C $\quad 4.981492-10.76552720 .100747$

O $\quad 4.669538-9.72959720 .739480$

O $\quad 4.140988-11.56303819 .559988$

O $\quad 2.294611-9.37625220 .099985$

H $\quad 1.702646-9.12761020 .825910$

H $\quad 3.262495-9.40619720 .468991$

O $\quad 1.289426-8.07155317 .779372$

H $\quad 1.900164-7.88616717 .029522$

H $\quad 1.797106-8.61107818 .418914$

C $\quad 0.036867-6.30688320 .286661$

H $\quad 0.461862-5.71123019 .467076$

H $\quad 0.864449-6.69133920 .897704$

N $\quad-0.680705-7.47333519 .701912$

H $\quad-0.150160-7.80921818 .874422$

H $\quad-0.783641-8.26539320 .419818$

H $\quad-1.637067-7.23049119 .382576$

h $\quad-0.184124-11.73087113 .951389$

h $\quad 0.229387-17.65902320 .992614$

h $\quad 7.828439-11.70977318 .294441$

h $\quad-0.605412-5.66647620 .891163$

TS9

ZPE at B1: 0.481979 (a.u.)

Total QM/MM energy at B2: -3166.538113 (a.u.)

Fe $\quad 2.029833-11.55778719 .963694$

P $\quad-0.525664-10.63083121 .703459$

C $\quad 0.861050-10.55216022 .861183$

C $\quad 1.689573-11.74093623 .138113$

O $\quad 2.347726-11.54574421 .903756$

C $\quad 2.605476-11.65518924 .344776$

O $\quad-1.515119-11.69223622 .186944$

O $\quad 0.092745-11.03128220 .308640$

O $\quad-1.060417-9.16795121 .633380$

H $\quad 1.277698-9.58219223 .156227$

H $\quad 2.014093-11.67220925 .274998$

H $\quad 3.306710-12.50064924 .372530$

H $\quad 3.194718-10.72532224 .306619$ 


\begin{tabular}{|c|c|c|c|}
\hline $\mathrm{H}$ & $1.090394-12.67205123 .152848$ & $\mathrm{H}$ & $-1.628957-7.17186019 .302752$ \\
\hline $\mathrm{C}$ & $-0.164584-10.87578014 .622344$ & $\mathrm{~h}$ & $-0.190192-11.69614913 .905123$ \\
\hline $\mathrm{H}$ & $-0.015912-9.91100814 .110017$ & $\mathrm{~h}$ & $0.187376-17.65019121 .011575$ \\
\hline $\mathrm{H}$ & $-1.181289-10.84277915 .040831$ & $\mathrm{~h}$ & $7.821678-11.68627318 .293445$ \\
\hline $\mathrm{N}$ & $2.166897-10.84501015 .664275$ & $\mathrm{~h}$ & $-0.596309-5.65040120 .862973$ \\
\hline $\mathrm{H}$ & $2.675872-10.56477314 .817967$ & & \\
\hline $\mathrm{C}$ & $0.802166-11.05224515 .754061$ & 10 & \\
\hline $\mathrm{C}$ & $2.710691-11.01405016 .886117$ & $\mathrm{ZPI}$ & at B1: 0.493356 (a.u.) \\
\hline $\mathrm{H}$ & $3.773405-10.89565717 .085742$ & Tot & QM/MM energy at B2: -3166.588693 (a.u.) \\
\hline $\mathrm{N}$ & $1.776698-11.34010217 .771683$ & $\mathrm{Fe}$ & $1.954803-11.61104919 .525132$ \\
\hline $\mathrm{C}$ & $0.583623-11.36236217 .078296$ & $\mathrm{P}$ & $-0.221676-10.78626721 .790972$ \\
\hline $\mathrm{H}$ & $-0.359746-11.57547217 .573445$ & $\mathrm{C}$ & $1.297261-10.94472722 .817576$ \\
\hline $\mathrm{C}$ & $1.194245-17.23956220 .936311$ & $\mathrm{C}$ & $1.287933-11.53317124 .169138$ \\
\hline $\mathrm{H}$ & $1.668760-17.23198621 .929503$ & $\mathrm{O}$ & $1.774656-12.28200723 .056574$ \\
\hline $\mathrm{H}$ & $1.739977-17.98478020 .333923$ & $\mathrm{C}$ & $2.287978-11.15022925 .227681$ \\
\hline $\mathrm{N}$ & $1.406898-15.69888818 .917379$ & $\mathrm{O}$ & $-1.326982-11.66181322 .370542$ \\
\hline $\mathrm{H}$ & $1.265366-16.39814818 .169501$ & $\mathrm{O}$ & $0.191285-11.27033920 .349385$ \\
\hline $\mathrm{C}$ & $1.328120-15.89156120 .287777$ & $\mathrm{O}$ & $-0.505210-9.24474821 .725511$ \\
\hline $\mathrm{C}$ & $1.653418-14.39778918 .673350$ & $\mathrm{H}$ & $2.104744-10.23242322 .588254$ \\
\hline $\mathrm{H}$ & $1.784645-13.99076917 .673946$ & $\mathrm{H}$ & $1.876049-10.35471825 .870258$ \\
\hline $\mathrm{N}$ & $1.741361-13.71701719 .807458$ & $\mathrm{H}$ & $2.517780-12.01209725 .875075$ \\
\hline $\mathrm{C}$ & $1.543342-14.63687220 .820735$ & $\mathrm{H}$ & $3.227536-10.79682324 .775867$ \\
\hline $\mathrm{H}$ & $1.594130-14.35478421 .869052$ & $\mathrm{H}$ & $0.319718-11.89563424 .534044$ \\
\hline $\mathrm{C}$ & $6.803914-11.78630018 .670563$ & $\mathrm{C}$ & $-0.262971-10.86214914 .308647$ \\
\hline $\mathrm{H}$ & $6.198160-11.39259317 .836811$ & $\mathrm{H}$ & $-0.142427-9.90955313 .768113$ \\
\hline $\mathrm{H}$ & $6.587482-12.86105818 .766292$ & $\mathrm{H}$ & $-1.281982-10.84826514 .721777$ \\
\hline $\mathrm{C}$ & $6.465758-11.01431719 .946313$ & $\mathrm{~N}$ & $2.064537-10.75635915 .348103$ \\
\hline $\mathrm{H}$ & $6.794318-11.55893220 .849364$ & $\mathrm{H}$ & $2.559941-10.44164914 .503775$ \\
\hline $\mathrm{H}$ & $6.966037-10.03430419 .987185$ & $\mathrm{C}$ & $0.706048-10.98875615 .443332$ \\
\hline $\mathrm{C}$ & $4.973729-10.72781020 .102760$ & $\mathrm{C}$ & $2.623435-10.93503016 .561112$ \\
\hline $\mathrm{O}$ & $4.662259-9.66990120 .704273$ & $\mathrm{H}$ & $3.687840-10.80625016 .752255$ \\
\hline $\mathrm{O}$ & $4.132822-11.55332919 .608778$ & $\mathrm{~N}$ & $1.699721-11.29069517 .443573$ \\
\hline $\mathrm{O}$ & $2.301581-9.31389020 .007653$ & $\mathrm{C}$ & $0.501373-11.32651816 .761882$ \\
\hline $\mathrm{H}$ & $1.718282-9.08679820 .744971$ & $\mathrm{H}$ & $-0.435235-11.57193217 .257419$ \\
\hline $\mathrm{H}$ & $3.265286-9.35314120 .388209$ & $\mathrm{C}$ & $1.171759-17.20512520 .949111$ \\
\hline $\mathrm{O}$ & $1.281334-8.04080417 .730728$ & $\mathrm{H}$ & $1.622705-17.13788321 .950965$ \\
\hline $\mathrm{H}$ & $1.886593-7.86619316 .974011$ & $\mathrm{H}$ & $1.750178-17.96621420 .398562$ \\
\hline $\mathrm{H}$ & $1.805388-8.54540718 .388130$ & $\mathrm{~N}$ & $1.396661-15.78974218 .852466$ \\
\hline $\mathrm{C}$ & $0.041150-6.27130520 .233536$ & $\mathrm{H}$ & $1.245676-16.52425118 .143777$ \\
\hline $\mathrm{H}$ & $0.443849-5.65689619 .417133$ & $\mathrm{C}$ & $1.299610-15.89093020 .231460$ \\
\hline $\mathrm{H}$ & $0.884245-6.66472620 .816271$ & $\mathrm{C}$ & $1.648803-14.50807918 .526488$ \\
\hline $\mathrm{N}$ & $-0.684043-7.42790019 .641735$ & $\mathrm{H}$ & $1.784218-14.17057817 .500909$ \\
\hline $\mathrm{H}$ & $-0.143430-7.77981018 .826490$ & $\mathrm{~N}$ & $1.729679-13.75748619 .612628$ \\
\hline $\mathrm{H}$ & $-0.819609-8.20291020 .368507$ & $\mathrm{C}$ & $1.511721-14.60270220 .686342$ \\
\hline
\end{tabular}


$6.670070-11.75546518 .682590$

H $\quad 6.065329-11.36574917 .846380$

H $\quad 6.450171-12.82888118 .783721$

C $\quad 6.319819-10.98119619 .953352$

H $\quad 6.687671-11.49415020 .860057$

H $\quad 6.778164-9.97971219 .972559$

C $\quad 4.816338-10.76178820 .131683$

O $4.453655-9.75869320 .791676$

O $\quad 4.018392-11.60220319 .589421$

O $\quad 2.114485-9.24029819 .915053$

H $\quad 1.435888-9.11124220 .599853$

H $\quad 3.010971-9.33147420 .375635$

O $\quad 1.263701-7.96299117 .624030$

H $\quad 1.862505-7.79536516 .861293$

H $\quad 1.785211-8.47287518 .279277$

C $\quad 0.102117-6.25220320 .239568$

H $\quad 0.460369-5.63874019 .402249$

H $\quad 0.970892-6.60842620 .805826$

$\mathrm{N} \quad-0.590295-7.44895519 .678893$

H $\quad-0.084350-7.74974918 .820689$

H $\quad-0.601666-8.24149320 .391851$

H $\quad-1.568411-7.24655619 .400890$

h $\quad-0.249395-11.71321213 .627784$

h $\quad 0.173180-17.63700621 .015330$

h $\quad 7.688838-11.66047018 .306885$

h $\quad-0.543077-5.64412620 .873628$

\section{TS10}

ZPE at B1: 0.485093 (a.u.)

Total QM/MM energy at B2: -3166.545672 (a.u.)

$\mathrm{Fe} \quad 2.123633-11.67309519 .982668$

P $\quad-0.408470-10.87399221 .725334$

C $\quad 0.709863-11.15669923 .087685$

C $\quad 2.154491-11.54265323 .039871$

O $2.613092-11.87650221 .774560$

C $\quad 3.063862-10.48108423 .703513$

O $\quad-1.698618-11.65151521 .989828$

O $\quad 0.215621-11.39269420 .366789$

O $\quad-0.548078-9.31012621 .621755$

H $\quad 0.309315-10.89489924 .076466$

H $\quad 2.769814-10.31334624 .752301$

H $4.107503-10.82276623 .674696$

H $\quad 3.012662-9.52740323 .156552$
$2.245827-12.45880423 .678794$

C $\quad-0.190853-10.92814814 .658986$

$\mathrm{H} \quad-0.055565-9.95684814 .155581$

H $\quad-1.205739-10.90944115 .082690$

N $\quad 2.142815-10.84860615 .684763$

H $\quad 2.636628-10.54828114 .835600$

C $\quad 0.786511-11.10143415 .783078$

C $\quad 2.703262-11.01064016 .898835$

H $\quad 3.762745-10.85974817 .092337$

N $\quad 1.787745-11.37622617 .789922$

C $\quad 0.590243-11.43628317 .105284$

H $\quad-0.340324-11.69222817 .604089$

C $\quad 1.232294-17.27859920 .901014$

H $\quad 1.715296-17.26482121 .889670$

H $\quad 1.759436-18.04283320 .305888$

N $\quad 1.440964-15.77268918 .856997$

$\mathrm{H} \quad 1.263401-16.47564018 .120324$

C $\quad 1.389651-15.94268120 .233297$

C $\quad 1.733357-14.48791018 .584531$

H $1.853661-14.10236617 .575060$

N $\quad 1.877092-13.79954219 .707653$

C $\quad 1.670068-14.69142420 .743367$

H $\quad 1.771475-14.37690421 .780124$

C $\quad 6.788540-11.72457818 .670763$

H $\quad 6.190254-11.36468817 .816997$

H $\quad 6.569299-12.79474018 .804784$

C $\quad 6.429408-10.90588319 .910224$

$\mathrm{H} \quad 6.754644-11.40865020 .838810$

H $\quad 6.915092-9.91729319 .919062$

C $\quad 4.929716-10.64718520 .047370$

O $\quad 4.566521-9.63615720 .688610$

O $\quad 4.132516-11.48315519 .487951$

O $2.086334-9.40924120 .146624$

H $\quad 1.401311-9.22369120 .820642$

H $\quad 3.023654-9.34131720 .530841$

O $\quad 1.271675-8.11458017 .755317$

H $\quad 1.897720-7.93115717 .017711$

$\mathrm{H} \quad 1.756157-8.64896618 .417401$

C $\quad 0.074238-6.27608420 .256662$

H $\quad 0.465943-5.66515419 .432122$

H $\quad 0.921797-6.64991720 .844500$

N $\quad-0.627403-7.45497819 .674691$

$\mathrm{H} \quad-0.101700-7.76707418 .831899$

H $\quad-0.663261-8.26026820 .373319$ 
H $\quad-1.596242-7.23772919 .376132$

h $\quad-0.212427-11.73991613 .931914$

h $\quad 0.219766-17.67230620 .989606$

h $\quad 7.809046-11.63875418 .297576$

h $\quad-0.570866-5.65283920 .875915$

\section{1}

ZPE at B1: 0.4854585 (a.u.)

Total QM/MM energy at B2: -3166.551457 (a.u.)

$\mathrm{Fe} \quad 2.234925-11.68222419 .976903$

P $\quad-0.295353-10.95057821 .743606$

C $\quad 0.702329-11.60032023 .078709$

C $\quad 2.200067-11.65994723 .045402$

O $2.711219-11.86959721 .769843$

C $\quad 2.827599-10.39636723 .682608$

O $\quad-1.720545-11.51993521 .847940$

O $\quad 0.313582-11.43552720 .358335$

O $\quad-0.152515-9.38806021 .801185$

H $\quad 0.208757-11.77005824 .042231$

H $\quad 2.543981-10.30628424 .743893$

H $\quad 3.922617-10.45735623 .601471$

H $\quad 2.487408-9.49478123 .151830$

$\mathrm{H} \quad 2.507502-12.51261523 .691965$

C $\quad-0.140648-10.92955914 .690130$

$\mathrm{H} \quad-0.006514-9.95471514 .193024$

H $\quad-1.148252-10.90670515 .130655$

N $\quad 2.210320-10.87263715 .678797$

H $\quad 2.692526-10.56239914 .826460$

C $\quad 0.854250-11.11806815 .795274$

C $\quad 2.787697-11.04564116 .883651$

H $\quad 3.850421-10.89936817 .062009$

$\mathrm{N} \quad 1.883700-11.41439117 .784430$

C $\quad 0.676655-11.46163217 .117406$

H $\quad-0.246909-11.72269117 .626790$

C $\quad 1.291376-17.29498820 .863200$

H $\quad 1.783554-17.29339921 .847685$

H $\quad 1.801693-18.06425120 .259579$

$\mathrm{N} \quad 1.505571-15.77711418 .827043$

H $\quad 1.313332-16.47278318 .087652$

C $\quad 1.463671-15.95768520 .202209$

C $\quad 1.818988-14.49427118 .564130$

H $1.935259-14.10211917 .557062$

N $\quad 1.982864-13.81831019 .691796$

C $\quad 1.766567-14.71499820 .719308$
H $\quad 1.868508-14.41514821 .758871$

C $\quad 6.879086-11.72461018 .655592$

H $\quad 6.270255-11.37793217 .803962$

H $\quad 6.657358-12.79158918 .811360$

C $\quad 6.538245-10.88103219 .882828$

H $\quad 6.896379-11.34829320 .817800$

H $\quad 7.004875-9.88360819 .850750$

C $\quad 5.039153-10.64219520 .045921$

O $\quad 4.678184-9.64008720 .702472$

O $\quad 4.240965-11.47573419 .486790$

O $\quad 2.215491-9.44139220 .124262$

H $\quad 1.551973-9.27662220 .831351$

H $\quad 3.162338-9.37761020 .494360$

O $\quad 1.194165-8.13179217 .872220$

H $\quad 1.797833-7.93543317 .119279$

H $\quad 1.714413-8.66561218 .510505$

C $\quad 0.019718-6.35423020 .379492$

H $\quad 0.441721-5.82306519 .515788$

$\mathrm{H} \quad 0.843770-6.69752721 .017241$

N $\quad-0.695233-7.56890419 .883884$

H $\quad-0.222736-7.88232519 .012615$

H $\quad-0.622326-8.35721820 .602391$

$\mathrm{H} \quad-1.693555-7.39540019 .661670$

h $\quad-0.180002-11.73405013 .955751$

h $\quad 0.273742-17.67298120 .961217$

h $\quad 7.895732-11.64408418 .270868$

h $\quad-0.621327-5.66879720 .933848$

\section{TS11}

ZPE at B1: 0.481505 (a.u.)

Total QM/MM energy at B2: -3166.539314 (a.u.)

$\mathrm{Fe} \quad 2.190886-11.68313819 .922135$

P $\quad-0.283764-10.95343621 .738851$

C $\quad 0.825967-11.73590722 .945772$

C $\quad 2.258817-11.39073223 .141088$

O $\quad 2.501013-11.89854421 .846947$

C $\quad 2.687465-9.95763223 .414613$

O $\quad-1.684350-11.54151121 .964742$

O $\quad 0.225664-11.39979520 .312026$

O $\quad-0.125019-9.40079121 .858326$

H $\quad 0.526315-12.74204423 .258203$

H $\quad 2.589834-9.74943524 .493123$

H $\quad 3.737961-9.82550523 .117094$

H $\quad 2.065054-9.23520422 .872461$ 


\begin{tabular}{|c|c|c|c|}
\hline $\mathrm{H}$ & $2.729658-12.05052923 .894609$ & $\mathrm{H}$ & $-1.681057-7.42369919 .675771$ \\
\hline $\mathrm{C}$ & $-0.135536-10.90497314 .663308$ & $\mathrm{~h}$ & $-0.182083-11.71531813 .935819$ \\
\hline $\mathrm{H}$ & $0.003911-9.93424914 .159684$ & $\mathrm{~h}$ & $0.260602-17.69496120 .967504$ \\
\hline $\mathrm{H}$ & $-1.141896-10.87264015 .106146$ & $\mathrm{~h}$ & $7.922057-11.62774618 .266606$ \\
\hline $\mathrm{N}$ & $2.221355-10.88702615 .645271$ & $\mathrm{~h}$ & $-0.613934-5.67222320 .926430$ \\
\hline $\mathrm{H}$ & $2.707561-10.58907814 .791056$ & & \\
\hline $\mathrm{C}$ & $0.859272-11.09834215 .767326$ & 12 & \\
\hline $\mathrm{C}$ & $2.797431-11.06846916 .849829$ & $\mathrm{ZPI}$ & at B1: 0.493153 (a.u.) \\
\hline $\mathrm{H}$ & $3.865039-10.95356417 .022781$ & Tot & QM/MM energy at B2: -3166.585067 (a.u.) \\
\hline $\mathrm{N}$ & $1.887132-11.40765117 .754541$ & $\mathrm{Fe}$ & $2.117409-11.65861119 .715248$ \\
\hline $\mathrm{C}$ & $0.677012-11.42644617 .092287$ & $\mathrm{P}$ & $-0.219788-10.93607121 .782696$ \\
\hline $\mathrm{H}$ & $-0.250706-11.65207717 .611164$ & $\mathrm{C}$ & $1.004087-11.78178422 .884419$ \\
\hline $\mathrm{C}$ & $1.279841-17.31962120 .876192$ & $\mathrm{C}$ & $2.211149-11.23832523 .546686$ \\
\hline $\mathrm{H}$ & $1.765575-17.31772921 .863864$ & $\mathrm{O}$ & $2.312097-11.94968222 .303764$ \\
\hline $\mathrm{H}$ & $1.792959-18.09022320 .276884$ & $\mathrm{C}$ & $2.632880-9.79374023 .584381$ \\
\hline $\mathrm{N}$ & $1.496323-15.79971418 .840645$ & $\mathrm{O}$ & $-1.594346-11.50655922 .137272$ \\
\hline $\mathrm{H}$ & $1.305287-16.49250218 .098484$ & $\mathrm{O}$ & $0.205672-11.40959620 .345298$ \\
\hline $\mathrm{C}$ & $1.456503-15.98300720 .215046$ & $\mathrm{O}$ & $-0.051085-9.38348521 .882692$ \\
\hline $\mathrm{C}$ & $1.805582-14.51477018 .580646$ & $\mathrm{H}$ & $0.589636-12.70961023 .306025$ \\
\hline $\mathrm{H}$ & $1.917955-14.12072517 .573459$ & $\mathrm{H}$ & $2.568282-9.42068424 .619343$ \\
\hline $\mathrm{N}$ & $1.967960-13.83892119 .708359$ & $\mathrm{H}$ & $3.674402-9.70221223 .240553$ \\
\hline $\mathrm{C}$ & $1.757211-14.74008420 .733372$ & $\mathrm{H}$ & $1.987893-9.17366522 .951066$ \\
\hline $\mathrm{H}$ & $1.866449-14.44091821 .772115$ & $\mathrm{H}$ & $2.589659-11.83893824 .388574$ \\
\hline $\mathrm{C}$ & $6.904788-11.70325818 .650700$ & $\mathrm{C}$ & $-0.198659-10.88894714 .486710$ \\
\hline $\mathrm{H}$ & $6.296923-11.35285817 .799899$ & $\mathrm{H}$ & $-0.072324-9.92634513 .964847$ \\
\hline $\mathrm{H}$ & $6.679048-12.76948218 .804893$ & $\mathrm{H}$ & $-1.209448-10.86714614 .919684$ \\
\hline $\mathrm{C}$ & $6.561303-10.86481219 .879248$ & $\mathrm{~N}$ & $2.150003-10.83464015 .481898$ \\
\hline $\mathrm{H}$ & $6.957975-11.31059620 .809085$ & $\mathrm{H}$ & $2.635586-10.52469514 .630722$ \\
\hline $\mathrm{H}$ & $6.992069-9.85223819 .828025$ & $\mathrm{C}$ & $0.790522-11.05093415 .600303$ \\
\hline $\mathrm{C}$ & $5.055788-10.68036620 .066008$ & $\mathrm{C}$ & $2.725354-11.02087116 .687629$ \\
\hline $\mathrm{O}$ & $4.675740-9.67758720 .724519$ & $\mathrm{H}$ & $3.794081-10.90594116 .859566$ \\
\hline $\mathrm{O}$ & $4.276338-11.53697719 .535147$ & $\mathrm{~N}$ & $1.815179-11.36666817 .587309$ \\
\hline $\mathrm{O}$ & $2.231190-9.46605120 .103184$ & $\mathrm{C}$ & $0.606641-11.38643616 .923586$ \\
\hline $\mathrm{H}$ & $1.571757-9.28561120 .806627$ & $\mathrm{H}$ & $-0.324319-11.61796517 .436188$ \\
\hline $\mathrm{H}$ & $3.182352-9.41013720 .474036$ & $\mathrm{C}$ & $1.275442-17.31929420 .887784$ \\
\hline $\mathrm{O}$ & $1.206104-8.11349017 .881257$ & $\mathrm{H}$ & $1.758181-17.31231421 .876976$ \\
\hline $\mathrm{H}$ & $1.799389-7.92388317 .117992$ & $\mathrm{H}$ & $1.792700-18.09056220 .292756$ \\
\hline $\mathrm{H}$ & $1.734642-8.65293618 .507610$ & $\mathrm{~N}$ & $1.473526-15.80942718 .846184$ \\
\hline $\mathrm{C}$ & $0.024951-6.35439220 .365593$ & $\mathrm{H}$ & $1.283651-16.50682018 .108835$ \\
\hline $\mathrm{H}$ & $0.418916-5.82880219 .485575$ & $\mathrm{C}$ & $1.446581-15.98482120 .221147$ \\
\hline $\mathrm{H}$ & $0.870064-6.67574920 .986846$ & $\mathrm{C}$ & $1.768605-14.52101818 .579273$ \\
\hline $\mathrm{N}$ & $-0.681520-7.58664619 .901462$ & $\mathrm{H}$ & $1.868314-14.13095317 .568618$ \\
\hline $\mathrm{H}$ & $-0.208596-7.91285719 .034577$ & $\mathrm{~N}$ & $1.937403-13.83637819 .698972$ \\
\hline $\mathrm{H}$ & $-0.602517-8.35858620 .633036$ & $\mathrm{C}$ & $1.743632-14.73502920 .729201$ \\
\hline
\end{tabular}




\begin{tabular}{|c|c|c|c|}
\hline $\mathrm{H}$ & $1.862948-14.43618621 .768091$ & $\mathrm{H}$ & $-2.489329-13.06050125 .017744$ \\
\hline $\mathrm{C}$ & $6.820992-11.72222018 .674762$ & $\mathrm{H}$ & $-2.449203-11.30883124 .691705$ \\
\hline $\mathrm{H}$ & $6.207728-11.35156917 .836285$ & $\mathrm{H}$ & $-4.594840-13.25614923 .636006$ \\
\hline $\mathrm{H}$ & $6.598236-12.79206418 .805087$ & $\mathrm{C}$ & $-5.301667-11.54755514 .684868$ \\
\hline $\mathrm{C}$ & $6.486091-10.91543819 .927779$ & $\mathrm{H}$ & $-4.970744-10.61357014 .199701$ \\
\hline $\mathrm{H}$ & $6.890246-11.38766720 .841106$ & $\mathrm{H}$ & $-6.285155-11.32927215 .128550$ \\
\hline $\mathrm{H}$ & $6.920249-9.90335819 .900339$ & $\mathrm{~N}$ & $-3.013547-12.09049915 .746551$ \\
\hline $\mathrm{C}$ & $4.982234-10.72734820 .136525$ & $\mathrm{H}$ & $-2.409191-12.04982814 .923418$ \\
\hline $\mathrm{O}$ & $4.613650-9.72549820 .796332$ & $\mathrm{C}$ & $-4.391387-11.94590815 .806155$ \\
\hline $\mathrm{O}$ & $4.194216-11.58954019 .619055$ & $\mathrm{C}$ & $-2.562794-12.34746316 .994890$ \\
\hline $\mathrm{O}$ & $2.177006-9.37397320 .076600$ & $\mathrm{H}$ & $-1.514873-12.49914317 .234294$ \\
\hline $\mathrm{H}$ & $1.515114-9.25820020 .792204$ & $\mathrm{~N}$ & $-3.562687-12.38382117 .863650$ \\
\hline $\mathrm{H}$ & $3.101981-9.38718920 .481108$ & $\mathrm{C}$ & $-4.704075-12.13146917 .135573$ \\
\hline $\mathrm{O}$ & $1.199962-8.07574817 .849654$ & $\mathrm{H}$ & $-5.678837-12.05966017 .614688$ \\
\hline $\mathrm{H}$ & $1.798808-7.88946717 .090755$ & $\mathrm{C}$ & $-4.430658-18.35044420 .891841$ \\
\hline $\mathrm{H}$ & $1.726364-8.59839718 .494619$ & $\mathrm{H}$ & $-4.007498-18.41190521 .905899$ \\
\hline $\mathrm{C}$ & $0.044321-6.33491220 .342067$ & $\mathrm{H}$ & $-3.913807-19.11274520 .285794$ \\
\hline $\mathrm{H}$ & $0.415964-5.80250119 .456589$ & $\mathrm{~N}$ & $-4.157482-16.73492218 .940294$ \\
\hline $\mathrm{H}$ & $0.905100-6.65112520 .943298$ & $\mathrm{H}$ & $-4.346666-17.39173118 .174369$ \\
\hline $\mathrm{N}$ & $-0.655114-7.57401419 .883954$ & $\mathrm{C}$ & $-4.190955-16.99177020 .305035$ \\
\hline $\mathrm{H}$ & $-0.183540-7.89517419 .012854$ & $\mathrm{C}$ & $-3.878844-15.42884118 .753506$ \\
\hline $\mathrm{H}$ & $-0.566481-8.33868020 .614121$ & $\mathrm{H}$ & $-3.809215-14.95925417 .774926$ \\
\hline $\mathrm{H}$ & $-1.657216-7.41970119 .664197$ & $\mathrm{~N}$ & $-3.720640-14.81610019 .917295$ \\
\hline $\mathrm{h}$ & $-0.217885-11.71902313 .780544$ & $\mathrm{C}$ & $-3.906402-15.77665620 .892352$ \\
\hline $\mathrm{h}$ & $0.256981-17.69735520 .976528$ & $\mathrm{H}$ & $-3.812337-15.53578921 .948893$ \\
\hline $\mathrm{h}$ & $7.836554-11.63774218 .288027$ & $\mathrm{C}$ & $1.054579-12.43449118 .831334$ \\
\hline \multirow[t]{2}{*}{$\mathrm{h}$} & $-0.594932-5.66357420 .915414$ & $\mathrm{H}$ & $0.422256-12.00913818 .036411$ \\
\hline & & $\mathrm{H}$ & $0.763120-13.48926618 .948761$ \\
\hline \multicolumn{2}{|l|}{$\mathbf{1}^{\prime}$} & $\mathrm{C}$ & $0.805353-11.66326020 .126186$ \\
\hline \multicolumn{2}{|c|}{ ZPE at B1: 0.495084 (a.u.) } & $\mathrm{H}$ & $1.441510-12.00642820 .960733$ \\
\hline \multicolumn{2}{|r|}{ Total QM/MM energy at B2: -3236.432944 (a.u.) } & $\mathrm{H}$ & $1.020090-10.58654620 .016552$ \\
\hline $\mathrm{Fe}$ & $-3.420772-12.69811320 .044176$ & $\mathrm{C}$ & $-0.643224-11.78763620 .598237$ \\
\hline $\mathrm{P}$ & $-5.829491-11.31595121 .713138$ & $\mathrm{O}$ & $-1.002402-11.17370921 .633839$ \\
\hline $\mathrm{C}$ & $-4.786282-11.16051023 .202837$ & $\mathrm{O}$ & $-1.408574-12.53883919 .917047$ \\
\hline $\mathrm{C}$ & $-3.922958-12.38916123 .512377$ & $\mathrm{O}$ & $-3.261556-9.10013220 .433243$ \\
\hline $\mathrm{O}$ & $-3.063821-12.74353922 .418929$ & $\mathrm{O}$ & $-2.784487-9.09393521 .792834$ \\
\hline $\mathrm{C}$ & $-3.114054-12.18350524 .792419$ & $\mathrm{H}$ & $-3.865975-9.88453620 .408070$ \\
\hline $\mathrm{O}$ & $-6.697536-12.58065721 .842711$ & $\mathrm{H}$ & $-2.056451-9.76090021 .766525$ \\
\hline $\mathrm{O}$ & $-4.829596-11.39643920 .479916$ & $\mathrm{C}$ & $-5.766585-6.59091320 .803757$ \\
\hline $\mathrm{O}$ & $-6.672281-10.01676121 .573881$ & $\mathrm{H}$ & $-5.128875-6.18617320 .007723$ \\
\hline $\mathrm{H}$ & $-4.147542-10.27345023 .064501$ & $\mathrm{H}$ & $-5.120942-7.00150421 .589549$ \\
\hline $\mathrm{H}$ & $-5.458502-10.96304224 .054820$ & $\mathrm{~N}$ & $-6.594053-7.70623520 .245858$ \\
\hline $\mathrm{H}$ & $-2.324546-12.08642322 .322907$ & $\mathrm{H}$ & $-6.512519-8.61582220 .800173$ \\
\hline $\mathrm{H}$ & $-3.782117-12.00619225 .650458$ & $\mathrm{H}$ & $-7.610710-7.48058920 .209385$ \\
\hline
\end{tabular}




$$
\begin{array}{cl}
\text { h } & -5.515196-12.31785913 .943853 \\
\text { h } & -5.471409-18.67429220 .896970 \\
\text { h } & 2.054466-12.41843018 .397704 \\
\text { h } & -6.394078-5.78607421 .186579
\end{array}
$$

\section{TS1'}

ZPE at B1: 0.481753 (a.u.)

Total QM/MM energy at B2: -3236.408202 (a.u.)

$\mathrm{Fe} \quad-3.250836-12.18976619 .980139$

$\mathrm{P} \quad-5.881906-11.34242221 .628815$

C $\quad-4.666079-11.05534722 .954311$

C $\quad-3.822237-12.28176823 .300733$

O $\quad-3.008100-12.68097922 .181882$

C $\quad-2.961633-12.03580124 .538195$

O $\quad-6.791922-12.51729222 .013776$

O $\quad-5.070343-11.69693220 .311840$

O $\quad-6.656154-10.00762421 .423816$

H $\quad-3.989615-10.22947322 .671319$

H $\quad-5.247401-10.74892223 .841327$

H $\quad-2.075681-12.29017022 .236297$

H $\quad-3.600850-11.80804125 .405850$

H $\quad-2.350687-12.91615324 .787787$

$\mathrm{H} \quad-2.288687-11.17986624 .369094$

H $\quad-4.504981-13.12934523 .490484$

C $\quad-5.250028-11.46830214 .671216$

H $\quad-4.959627-10.53448214 .160057$

H $\quad-6.228788-11.26868115 .133707$

N $\quad-2.920668-11.93400015 .694846$

H $\quad-2.336072-11.95098914 .855876$

C $\quad-4.300971-11.80052715 .782531$

C $\quad-2.428586-12.09266716 .940381$

H $\quad-1.372613-12.21071217 .160676$

N $\quad-3.407059-12.07195017 .835036$

C $\quad-4.576467-11.88940217 .128889$

H $\quad-5.533142-11.80596017 .640047$

C $\quad-4.283012-17.95121820 .856240$

$\mathrm{H} \quad-3.854199-17.93563321 .869827$

H $\quad-3.693822-18.68521120 .278992$

N $\quad-4.158184-16.37746118 .847343$

$\mathrm{H} \quad-4.380232-17.05859218 .113590$

C $\quad-4.135676-16.59709620 .219558$

C $\quad-3.863488-15.07802918 .615902$

H $\quad-3.839087-14.64320217 .619855$
$-3.640162-14.43634919 .749597$

C $\quad-3.801484-15.36815820 .756051$

H $\quad-3.641738-15.09564221 .796154$

C $\quad 1.233624-12.59064418 .794055$

H $0.568383-12.13295418 .046592$

H $\quad 0.972771-13.65932318 .849026$

C $\quad 1.022484-11.90768120 .140439$

$\mathrm{H} \quad 1.714816-12.25598420 .924185$

$\mathrm{H} \quad 1.185208-10.81740420 .068058$

C $\quad-0.387492-12.09453420 .685017$

O $\quad-0.620009-11.75436721 .866564$

O $\quad-1.264858-12.59198519 .901109$

O $\quad-2.659794-10.24886520 .253042$

O $\quad-2.167227-9.29223821 .865180$

H $\quad-3.456586-9.70452620 .175840$

H $\quad-1.521311-10.00870522 .017946$

C $\quad-5.848495-6.59370720 .767722$

H $\quad-5.191946-6.18387219 .989067$

H $\quad-5.227814-7.02649721 .563415$

N $\quad-6.689737-7.68149820 .178777$

H $\quad-6.586043-8.61726120 .689987$

H $\quad-7.709036-7.46085220 .189061$

H $\quad-6.462914-7.86220419 .179490$

h $\quad-5.462875-12.25253313 .944756$

h $\quad-5.282484-18.38536720 .881487$

h $\quad 2.223960-12.52254218 .343872$

h $\quad-6.459780-5.78064921 .159300$

$2^{\prime}$

ZPE at B1: 0.485705 (a.u.)

Total QM/MM energy at B2: -3236.417023 (a.u.)

Fe $\quad-3.238223-12.16943120 .006687$

P $\quad-5.943234-11.41331121 .602478$

C $\quad-4.782520-11.12532622 .978811$

C $\quad-3.808057-12.25847523 .286467$

O $\quad-2.988423-12.56817222 .147790$

C $\quad-2.941346-11.92536324 .498583$

O $\quad-6.861561-12.58331121 .981600$

O $\quad-5.101292-11.78880020 .315283$

O $\quad-6.702905-10.07444121 .385053$

H $\quad-4.217449-10.18581922 .792662$

$\mathrm{H} \quad-5.409096-10.92912123 .865646$

$\mathrm{H} \quad-2.018469-12.23007322 .227102$

$\mathrm{H} \quad-3.575366-11.75165825 .382430$ 


\begin{tabular}{|c|c|c|c|}
\hline $\mathrm{H}$ & $-2.245402-12.74402024 .733763$ & $\mathrm{H}$ & $-6.456572-7.89495919 .177941$ \\
\hline $\mathrm{H}$ & $-2.363800-11.00906824 .296200$ & $\mathrm{~h}$ & $-5.468406-12.27186813 .958084$ \\
\hline $\mathrm{H}$ & $-4.406575-13.16650023 .492443$ & $\mathrm{~h}$ & $-5.291702-18.39780020 .912503$ \\
\hline $\mathrm{C}$ & $-5.253523-11.49228614 .688935$ & $\mathrm{~h}$ & $2.221619-12.54199418 .341859$ \\
\hline $\mathrm{H}$ & $-4.956358-10.55707914 .184350$ & $\mathrm{~h}$ & $-6.437886-5.81074321 .158591$ \\
\hline $\mathrm{H}$ & $-6.232152-11.29028215 .150601$ & & \\
\hline $\mathrm{N}$ & $-2.929252-11.98473815 .712931$ & \multicolumn{2}{|c|}{ TS2' } \\
\hline $\mathrm{H}$ & $-2.343781-11.99671914 .874206$ & \multicolumn{2}{|c|}{ ZPE at B1: 0.480919 (a.u.) } \\
\hline $\mathrm{C}$ & $-4.308605-11.83912115 .799503$ & \multicolumn{2}{|c|}{ Total QM/MM energy at B2: -3236.414319 (a.u.) } \\
\hline $\mathrm{C}$ & $-2.440481-12.15699216 .957504$ & $\mathrm{Fe}$ & $-3.230489-12.15116519 .994827$ \\
\hline $\mathrm{H}$ & $-1.386894-12.29053217 .180925$ & $\mathrm{P}$ & $-5.933497-11.38819821 .600573$ \\
\hline $\mathrm{N}$ & $-3.420901-12.13536517 .850643$ & $\mathrm{C}$ & $-4.764865-11.09150322 .965669$ \\
\hline $\mathrm{C}$ & $-4.587661-11.93579417 .144414$ & $\mathrm{C}$ & $-3.773842-12.19661423 .287752$ \\
\hline $\mathrm{H}$ & $-5.544073-11.85045417 .655771$ & $\mathrm{O}$ & $-2.975946-12.53829822 .146134$ \\
\hline $\mathrm{C}$ & $-4.292223-17.96302920 .903365$ & $\mathrm{C}$ & $-2.893680-11.83284524 .482180$ \\
\hline $\mathrm{H}$ & $-3.877591-17.95267321 .922931$ & $\mathrm{O}$ & $-6.831757-12.56660922 .003609$ \\
\hline $\mathrm{H}$ & $-3.695151-18.69483120 .331506$ & $\mathrm{O}$ & $-5.097022-11.76143020 .313696$ \\
\hline $\mathrm{N}$ & $-4.133572-16.38894018 .900919$ & $\mathrm{O}$ & $-6.715428-10.06303521 .392322$ \\
\hline $\mathrm{H}$ & $-4.347516-17.06925718 .164802$ & $\mathrm{H}$ & $-4.155781-10.08119422 .723516$ \\
\hline $\mathrm{C}$ & $-4.135306-16.60701020 .273646$ & $\mathrm{H}$ & $-5.349597-10.82139623 .861113$ \\
\hline $\mathrm{C}$ & $-3.833029-15.08984418 .673863$ & $\mathrm{H}$ & $-1.999564-12.20684622 .211365$ \\
\hline $\mathrm{H}$ & $-3.790501-14.65871217 .677343$ & $\mathrm{H}$ & $-3.519347-11.65504625 .371270$ \\
\hline $\mathrm{N}$ & $-3.630204-14.44581319 .810184$ & $\mathrm{H}$ & $-2.187965-12.64221024 .719045$ \\
\hline $\mathrm{C}$ & $-3.810970-15.37608820 .813910$ & $\mathrm{H}$ & $-2.332973-10.91252424 .257335$ \\
\hline $\mathrm{H}$ & $-3.673859-15.10685621 .858349$ & $\mathrm{H}$ & $-4.369520-13.09918223 .532623$ \\
\hline $\mathrm{C}$ & $1.232569-12.61638718 .793868$ & $\mathrm{C}$ & $-5.260623-11.49164914 .681412$ \\
\hline $\mathrm{H}$ & $0.566740-12.13685318 .060148$ & $\mathrm{H}$ & $-4.969310-10.55526214 .175686$ \\
\hline $\mathrm{H}$ & $0.967977-13.68519318 .823958$ & $\mathrm{H}$ & $-6.239438-11.29475915 .144804$ \\
\hline $\mathrm{C}$ & $1.033860-11.96772920 .158372$ & $\mathrm{~N}$ & $-2.932874-11.97209315 .702303$ \\
\hline $\mathrm{H}$ & $1.722414-12.34992020 .929144$ & $\mathrm{H}$ & $-2.348501-11.98280814 .863017$ \\
\hline $\mathrm{H}$ & $1.220232-10.87997720 .116455$ & $\mathrm{C}$ & $-4.312656-11.83333615 .790754$ \\
\hline $\mathrm{C}$ & $-0.376323-12.14226720 .706844$ & $\mathrm{C}$ & $-2.441364-12.14291416 .945967$ \\
\hline $\mathrm{O}$ & $-0.595396-11.82329921 .890132$ & $\mathrm{H}$ & $-1.386030-12.26945417 .165625$ \\
\hline $\mathrm{O}$ & $-1.268171-12.60875619 .911924$ & $\mathrm{~N}$ & $-3.420611-12.12588717 .840346$ \\
\hline $\mathrm{O}$ & $-2.763767-10.29500620 .141468$ & $\mathrm{C}$ & $-4.589468-11.93245517 .135955$ \\
\hline $\mathrm{O}$ & $-2.940570-8.98095922 .461443$ & $\mathrm{H}$ & $-5.545884-11.85120517 .648081$ \\
\hline $\mathrm{H}$ & $-3.472673-9.79241819 .711916$ & $\mathrm{C}$ & $-4.277893-17.94678320 .906831$ \\
\hline $\mathrm{H}$ & $-2.746388-9.47397421 .601213$ & $\mathrm{H}$ & $-3.862353-17.93339521 .926014$ \\
\hline $\mathrm{C}$ & $-5.825110-6.61666420 .754779$ & $\mathrm{H}$ & $-3.677450-18.67625220 .335428$ \\
\hline $\mathrm{H}$ & $-5.200496-6.19422819 .956497$ & $\mathrm{~N}$ & $-4.130714-16.37314618 .903199$ \\
\hline $\mathrm{H}$ & $-5.169954-7.05449621 .520248$ & $\mathrm{H}$ & $-4.344176-17.05530418 .168662$ \\
\hline $\mathrm{N}$ & $-6.674726-7.70917520 .178116$ & $\mathrm{C}$ & $-4.127086-16.59035020 .275694$ \\
\hline $\mathrm{H}$ & $-6.576726-8.64310020 .687659$ & $\mathrm{C}$ & $-3.835122-15.07333618 .674118$ \\
\hline $\mathrm{H}$ & $-7.693418-7.48453120 .191358$ & $\mathrm{H}$ & $-3.796844-14.64289817 .677097$ \\
\hline
\end{tabular}




\begin{tabular}{|c|c|c|c|}
\hline $\mathrm{N}$ & $-3.630315-14.42773519 .809215$ & $\mathrm{H}$ & $-1.905846-12.08389724 .551845$ \\
\hline $\mathrm{C}$ & $-3.805373-15.35794120 .814323$ & $\mathrm{H}$ & $-2.487314-10.49581023 .942539$ \\
\hline $\mathrm{H}$ & $-3.664814-15.08727821 .858083$ & $\mathrm{H}$ & $-4.027137-13.14505123 .728485$ \\
\hline $\mathrm{C}$ & $1.242464-12.62003518 .789088$ & $\mathrm{C}$ & $-5.303526-11.52053614 .667292$ \\
\hline $\mathrm{H}$ & $0.575194-12.14429918 .054369$ & $\mathrm{H}$ & $-5.026978-10.57670814 .166992$ \\
\hline $\mathrm{H}$ & $0.980711-13.68947318 .822114$ & $\mathrm{H}$ & $-6.285750-11.34201715 .130785$ \\
\hline $\mathrm{C}$ & $1.043611-11.96689720 .151139$ & $\mathrm{~N}$ & $-2.965443-11.95663115 .680951$ \\
\hline $\mathrm{H}$ & $1.730637-12.34721320 .924306$ & $\mathrm{H}$ & $-2.385088-11.95120914 .839580$ \\
\hline $\mathrm{H}$ & $1.230685-10.87937120 .105763$ & $\mathrm{C}$ & $-4.348212-11.85239115 .773474$ \\
\hline $\mathrm{C}$ & $-0.366920-12.13939620 .699328$ & $\mathrm{C}$ & $-2.466053-12.12135916 .922491$ \\
\hline $\mathrm{O}$ & $-0.582683-11.82269121 .884341$ & $\mathrm{H}$ & $-1.406417-12.21254417 .139836$ \\
\hline $\mathrm{O}$ & $-1.261044-12.60555919 .907502$ & $\mathrm{~N}$ & $-3.443405-12.13642717 .818696$ \\
\hline $\mathrm{O}$ & $-2.752785-10.28638320 .105817$ & $\mathrm{C}$ & $-4.618959-11.96790317 .118611$ \\
\hline $\mathrm{O}$ & $-3.267132-9.09030522 .484578$ & $\mathrm{H}$ & $-5.575515-11.91330517 .634225$ \\
\hline $\mathrm{H}$ & $-3.444935-9.78216819 .650996$ & $\mathrm{C}$ & $-4.270568-17.95711720 .905485$ \\
\hline $\mathrm{H}$ & $-2.936483-9.50893021 .637019$ & $\mathrm{H}$ & $-3.857496-17.94274121 .925680$ \\
\hline $\mathrm{C}$ & $-5.827313-6.61377520 .762742$ & $\mathrm{H}$ & $-3.669485-18.68871420 .337582$ \\
\hline $\mathrm{H}$ & $-5.194489-6.19354319 .969750$ & $\mathrm{~N}$ & $-4.103596-16.38811218 .899994$ \\
\hline $\mathrm{H}$ & $-5.181861-7.06010421 .531430$ & $\mathrm{H}$ & $-4.310524-17.07124818 .164442$ \\
\hline $\mathrm{N}$ & $-6.677880-7.70097720 .177302$ & $\mathrm{C}$ & $-4.114261-16.60198520 .273306$ \\
\hline $\mathrm{H}$ & $-6.583867-8.63341320 .688175$ & $\mathrm{C}$ & $-3.799044-15.08975718 .671716$ \\
\hline $\mathrm{H}$ & $-7.696052-7.47449820 .186579$ & $\mathrm{H}$ & $-3.746149-14.66099217 .674613$ \\
\hline $\mathrm{H}$ & $-6.455298-7.88599419 .178380$ & $\mathrm{~N}$ & $-3.605716-14.44201019 .807397$ \\
\hline $\mathrm{h}$ & $-5.472540-12.27254613 .951099$ & $\mathrm{C}$ & $-3.793820-15.36896820 .812268$ \\
\hline $\mathrm{h}$ & $-5.275351-18.38616220 .916325$ & $\mathrm{H}$ & $-3.666308-15.09858921 .858045$ \\
\hline $\mathrm{h}$ & $2.231682-12.54410418 .337703$ & $\mathrm{C}$ & $1.261765-12.64630718 .767435$ \\
\hline \multirow[t]{2}{*}{$\mathrm{h}$} & $-6.440116-5.80624921 .163293$ & $\mathrm{H}$ & $0.589052-12.19162118 .024539$ \\
\hline & & $\mathrm{H}$ & $1.016597-13.71928318 .813806$ \\
\hline $3^{\prime}$ & & $\mathrm{C}$ & $1.052283-11.97677020 .118815$ \\
\hline \multicolumn{2}{|c|}{ ZPE at B1: 0.485226 (a.u.) } & $\mathrm{H}$ & $1.739349-12.33956520 .900323$ \\
\hline \multicolumn{2}{|r|}{ Total QM/MM energy at B2: -3236.439153 (a.u.) } & $\mathrm{H}$ & $1.228024-10.88787320 .059167$ \\
\hline $\mathrm{Fe}$ & $-3.239610-12.14688319 .975206$ & $\mathrm{C}$ & $-0.358197-12.15379320 .663652$ \\
\hline $\mathrm{P}$ & $-5.987129-11.48461621 .577001$ & $\mathrm{O}$ & $-0.567068-11.84519021 .854661$ \\
\hline $\mathrm{C}$ & $-4.920575-11.37988923 .013456$ & $\mathrm{O}$ & $-1.256248-12.60767019 .873463$ \\
\hline $\mathrm{C}$ & $-3.688540-12.17447123 .294778$ & $\mathrm{O}$ & $-2.766559-10.28974220 .014206$ \\
\hline $\mathrm{O}$ & $-2.957966-12.49297122 .105543$ & $\mathrm{O}$ & $-3.400114-8.85305222 .231293$ \\
\hline $\mathrm{C}$ & $-2.795912-11.47874524 .328917$ & $\mathrm{H}$ & $-3.371458-9.80713019 .428337$ \\
\hline $\mathrm{O}$ & $-6.996189-12.61494321 .834000$ & $\mathrm{H}$ & $-3.010755-9.30765521 .443572$ \\
\hline $\mathrm{O}$ & $-5.113863-11.82763720 .310648$ & $\mathrm{C}$ & $-5.782954-6.58998620 .754630$ \\
\hline $\mathrm{O}$ & $-6.656518-10.08794721 .440875$ & $\mathrm{H}$ & $-5.194131-6.15703319 .935034$ \\
\hline $\mathrm{H}$ & $-4.166162-9.42662822 .392785$ & $\mathrm{H}$ & $-5.090747-7.01554921 .493427$ \\
\hline $\mathrm{H}$ & $-5.385190-10.90482123 .887229$ & $\mathrm{~N}$ & $-6.625694-7.70333720 .202020$ \\
\hline $\mathrm{H}$ & $-1.958767-12.20445022 .157952$ & $\mathrm{H}$ & $-6.510553-8.62539920 .718059$ \\
\hline $\mathrm{H}$ & $-3.356218-11.33464325 .266549$ & $\mathrm{H}$ & $-7.647063-7.49104520 .216380$ \\
\hline
\end{tabular}


H $\quad-6.411880-7.90070319 .203129$

h $\quad-5.502135-12.29992813 .931649$

h $\quad-5.268775-18.39481220 .914053$

h $\quad 2.251926-12.55911618 .320170$

h $\quad-6.404603-5.79720921 .170710$

TS3' $^{\prime}$

ZPE at B1: 0.478709 (a.u.)

Total QM/MM energy at B2: -3236.398479 (a.u.)

$\mathrm{Fe} \quad-3.277690-12.00648119 .938203$

$\mathrm{P} \quad-5.949896-11.18160821 .489238$

C $\quad-4.765887-10.91092322 .859562$

C $\quad-3.917575-12.13156123 .218341$

O $\quad-3.072839-12.51870822 .118456$

C $\quad-3.085779-11.89782924 .477689$

O $\quad-6.906976-12.30204321 .917310$

O $\quad-5.126815-11.69096620 .227476$

O $\quad-6.609892-9.80463421 .189929$

H $\quad-4.103998-10.06185222 .635031$

H $\quad-5.384950-10.63152923 .729405$

H $\quad-2.124316-12.18076322 .209295$

H $\quad-3.744473-11.69258125 .336196$

H $\quad-2.470039-12.77661324 .723118$

H $\quad-2.417819-11.03233024 .339174$

H $\quad-4.602324-12.98209823 .383553$

C $\quad-5.293296-11.44855414 .655396$

H $\quad-5.037073-10.51286614 .129764$

H $\quad-6.269904-11.27138815 .130674$

N $\quad-2.942511-11.86999515 .661275$

$\mathrm{H} \quad-2.366294-11.90821214 .817107$

C $\quad-4.322764-11.73533415 .760926$

C $\quad-2.434750-11.99081316 .903152$

H $\quad-1.376452-12.11031717 .112146$

N $\quad-3.403949-11.94265617 .808242$

C $\quad-4.583231-11.78027917 .111958$

H $\quad-5.533342-11.68617917 .632698$

C $\quad-4.233831-17.81886120 .859841$

H $\quad-3.802171-17.79649221 .872173$

H $\quad-3.633420-18.54224420 .280607$

N $\quad-4.139235-16.24395618 .852617$

H $\quad-4.353752-16.93138018 .123634$

C $\quad-4.111080-16.46145920 .224210$

C $\quad-3.866435-14.93877318 .620233$

H $\quad-3.849974-14.50822717 .622360$
$\mathrm{N}$

$-3.645548-14.95173921 .799787$

C $\quad 1.225861-12.63449218 .807875$

H $\quad 0.550193-12.20207518 .055122$

$\mathrm{H} \quad 0.980060-13.70545418 .881051$

C $\quad 1.013879-11.93084120 .143084$

H $\quad 1.689332-12.28654020 .937909$

H $\quad 1.206557-10.84597920 .059629$

C $\quad-0.408506-12.07142620 .668570$

O $\quad-0.642457-11.70448221 .847204$

O $\quad-1.287551-12.54357419 .879871$

O $\quad-2.799041-10.23424320 .210675$

O $\quad-1.949866-9.21931022 .034108$

$\mathrm{H} \quad-2.722766-9.69473021 .150159$

$\mathrm{H} \quad-1.340462-9.98179922 .148501$

C $\quad-5.372053-6.53759120 .539610$

H $\quad-4.649196-5.97404919 .934873$

H $\quad-4.842404-6.99166821 .387343$

N $\quad-5.913128-7.64686719 .695853$

H $\quad-6.278431-8.46939720 .277762$

H $\quad-6.635033-7.28708819 .044014$

H $\quad-5.167320-8.03805719 .096625$

h $\quad-5.492420-12.24448813 .937828$

h $\quad-5.224637-18.27226320 .888105$

h $\quad 2.213123-12.55428518 .352961$

h $\quad-6.123943-5.83354420 .896045$

$4^{\prime}$

ZPE at B1: 0.482061 (a.u.)

Total QM/MM energy at B2: -3236.434016 (a.u.)

Fe $\quad-3.292909-12.11145819 .923424$

P $\quad-5.904559-11.24227421 .473042$

C $\quad-4.718642-10.94141722 .831771$

C $\quad-3.913391-12.16691423 .263168$

O $\quad-3.059073-12.62270722 .203115$

C $\quad-3.105364-11.89546324 .531648$

O $\quad-6.916168-12.29382021 .948093$

O $\quad-5.123154-11.91620820 .250273$

O $\quad-6.497944-9.85470221 .098489$

H $\quad-4.025414-10.12786022 .573521$

H $\quad-5.339820-10.59579823 .676376$

$\mathrm{H} \quad-2.156729-12.18472522 .239245$

H $\quad-3.778195-11.65075425 .368728$ 


\begin{tabular}{|c|c|c|c|}
\hline $\mathrm{H}$ & $-2.505822-12.77247524 .820635$ & $\mathrm{H}$ & $-5.123440-7.99019019 .002858$ \\
\hline $\mathrm{H}$ & $-2.426252-11.04163724 .375240$ & $\mathrm{~h}$ & $-5.513102-12.25514713 .898056$ \\
\hline $\mathrm{H}$ & $-4.621339-12.99347723 .450320$ & $\mathrm{~h}$ & $-5.214640-18.25244920 .896227$ \\
\hline $\mathrm{C}$ & $-5.315755-11.45889914 .615766$ & $\mathrm{~h}$ & $2.167110-12.55707418 .348629$ \\
\hline $\mathrm{H}$ & $-5.060965-10.52167614 .092172$ & $\mathrm{~h}$ & $-6.090887-5.84098520 .864529$ \\
\hline $\mathrm{H}$ & $-6.291487-11.28521515 .093900$ & & \\
\hline $\mathrm{N}$ & $-2.964553-11.89495215 .606209$ & \multicolumn{2}{|c|}{ TS4' } \\
\hline $\mathrm{H}$ & $-2.392691-11.91823514 .759170$ & \multicolumn{2}{|c|}{ ZPE at B1: 0.486016 (a.u.) } \\
\hline $\mathrm{C}$ & $-4.342389-11.75969415 .714711$ & \multicolumn{2}{|c|}{ Total QM/MM energy at B2: -3236.427105 (a.u.) } \\
\hline $\mathrm{C}$ & $-2.455347-12.05905016 .845995$ & $\mathrm{Fe}$ & $-3.243279-12.22761120 .125238$ \\
\hline $\mathrm{H}$ & $-1.396431-12.19025217 .045232$ & $\mathrm{P}$ & $-5.960203-11.55456121 .625798$ \\
\hline $\mathrm{N}$ & $-3.420027-12.03793017 .752180$ & $\mathrm{C}$ & $-4.879073-11.58883623 .045636$ \\
\hline $\mathrm{C}$ & $-4.598284-11.84749217 .065904$ & $\mathrm{C}$ & $-3.486569-12.12837723 .170338$ \\
\hline $\mathrm{H}$ & $-5.548377-11.76654517 .589527$ & $\mathrm{O}$ & $-2.861688-12.46946921 .970093$ \\
\hline $\mathrm{C}$ & $-4.228323-17.78922620 .870319$ & $\mathrm{C}$ & $-2.626119-11.13721623 .985109$ \\
\hline $\mathrm{H}$ & $-3.802959-17.75468621 .884958$ & $\mathrm{O}$ & $-7.056351-12.61448221 .817566$ \\
\hline $\mathrm{H}$ & $-3.617636-18.51223120 .301238$ & $\mathrm{O}$ & $-5.125222-11.89247320 .329695$ \\
\hline $\mathrm{N}$ & $-4.138454-16.23257418 .848820$ & $\mathrm{O}$ & $-6.528902-10.09976221 .537994$ \\
\hline $\mathrm{H}$ & $-4.357655-16.92512318 .126093$ & $\mathrm{H}$ & $-4.744039-9.22390921 .883866$ \\
\hline $\mathrm{C}$ & $-4.109474-16.43641120 .223100$ & $\mathrm{H}$ & $-5.324683-11.20473923 .971786$ \\
\hline $\mathrm{C}$ & $-3.859570-14.93379718 .599901$ & $\mathrm{H}$ & $-1.373320-11.11125121 .441571$ \\
\hline $\mathrm{H}$ & $-3.844814-14.51310217 .598809$ & $\mathrm{H}$ & $-3.031027-11.02037725 .004311$ \\
\hline $\mathrm{N}$ & $-3.640308-14.27674819 .726544$ & $\mathrm{H}$ & $-1.593967-11.50699924 .060564$ \\
\hline $\mathrm{C}$ & $-3.790204-15.19777120 .746198$ & $\mathrm{H}$ & $-2.640622-10.15530223 .487292$ \\
\hline $\mathrm{H}$ & $-3.627866-14.90775521 .780635$ & $\mathrm{H}$ & $-3.570965-13.05736223 .790068$ \\
\hline $\mathrm{C}$ & $1.175294-12.64130918 .792783$ & $\mathrm{C}$ & $-5.234001-11.49810014 .735649$ \\
\hline $\mathrm{H}$ & $0.502810-12.23104018 .024732$ & $\mathrm{H}$ & $-4.943527-10.55748614 .236186$ \\
\hline $\mathrm{H}$ & $0.939817-13.71287218 .886497$ & $\mathrm{H}$ & $-6.207755-11.30139015 .209790$ \\
\hline $\mathrm{C}$ & $0.948406-11.90783120 .108738$ & $\mathrm{~N}$ & $-2.905217-12.04248715 .713058$ \\
\hline $\mathrm{H}$ & $1.623975-12.23913520 .914299$ & $\mathrm{H}$ & $-2.350277-12.09237914 .857245$ \\
\hline $\mathrm{H}$ & $1.133341-10.82356520 .001309$ & $\mathrm{C}$ & $-4.275893-11.85523915 .831927$ \\
\hline $\mathrm{C}$ & $-0.471216-12.03663020 .647310$ & $\mathrm{C}$ & $-2.394740-12.22304516 .951754$ \\
\hline $\mathrm{O}$ & $-0.704711-11.61573721 .798414$ & $\mathrm{H}$ & $-1.341929-12.40717017 .143853$ \\
\hline $\mathrm{O}$ & $-1.347024-12.57164919 .880170$ & $\mathrm{~N}$ & $-3.348021-12.15850017 .869481$ \\
\hline $\mathrm{O}$ & $-2.967040-10.52911720 .100643$ & $\mathrm{C}$ & $-4.522129-11.92983317 .186003$ \\
\hline $\mathrm{O}$ & $-1.937936-9.06325622 .184102$ & $\mathrm{H}$ & $-5.462708-11.81017317 .719858$ \\
\hline $\mathrm{H}$ & $-2.428097-9.46299521 .438706$ & $\mathrm{C}$ & $-4.237074-17.97999220 .899609$ \\
\hline $\mathrm{H}$ & $-1.300455-9.77585122 .362917$ & $\mathrm{H}$ & $-3.821670-17.97647421 .918890$ \\
\hline $\mathrm{C}$ & $-5.332454-6.53524920 .502806$ & $\mathrm{H}$ & $-3.646252-18.71515920 .325329$ \\
\hline $\mathrm{H}$ & $-4.615082-5.95803419 .904724$ & $\mathrm{~N}$ & $-4.067990-16.40059418 .902498$ \\
\hline $\mathrm{H}$ & $-4.796841-6.99205121 .345587$ & $\mathrm{H}$ & $-4.295977-17.07661518 .166736$ \\
\hline $\mathrm{N}$ & $-5.860998-7.64156419 .643643$ & $\mathrm{C}$ & $-4.064878-16.62307120 .275498$ \\
\hline $\mathrm{H}$ & $-6.187823-8.48882820 .210117$ & $\mathrm{C}$ & $-3.743019-15.10688818 .677368$ \\
\hline $\mathrm{H}$ & $-6.611620-7.28755219 .022271$ & $\mathrm{H}$ & $-3.701148-14.67018117 .683480$ \\
\hline
\end{tabular}




$$
\begin{aligned}
& \mathrm{N} \quad-3.520264-14.47189719 .815021 \\
& \text { C } \quad-3.713988-15.40053720 .817818 \\
& \mathrm{H} \quad-3.571895-15.13263321 .862573 \\
& \text { C } \quad 1.544013-12.65351618 .618945 \\
& \mathrm{H} \quad 0.889302-12.21593717 .849136 \\
& \text { H } \quad 1.286796-13.72096918 .694894 \\
& \text { C } \quad 1.321101-11.93075619 .935638 \\
& \text { H } \quad 1.913552-12.34626320 .768953 \\
& \text { H } \quad 1.606069-10.86709519 .881596 \\
& \text { C } \quad-0.128568-11.96395320 .344019 \\
& \text { O } \quad-0.386842-11.18918521 .367178 \\
& \text { O } \quad-0.976840-12.62569919 .748186 \\
& \text { O } \quad-2.608613-10.38507620 .120718 \\
& \text { O } \quad-3.878467-8.77908021 .864972 \\
& \text { H } \quad-2.309167-10.08863019 .242413 \\
& \mathrm{H} \quad-3.399562-9.28139721 .157033 \\
& \text { C } \quad-5.823720-6.55680320 .729190 \\
& \text { H } \quad-5.280088-6.11203419 .885319 \\
& \mathrm{H} \quad-5.091495-6.97016021 .432273 \\
& \text { N } \quad-6.654882-7.69421520 .205672 \\
& \text { H } \quad-6.523351-8.59767620 .735679 \\
& \text { H } \quad-7.678032-7.49218120 .211137 \\
& \text { H } \quad-6.428825-7.91433519 .213687 \\
& \text { h } \quad-5.454433-12.26911513 .997400 \\
& \text { h } \quad-5.239808-18.40720120 .908749 \\
& \text { h } \quad 2.542904-12.57655718 .189574 \\
& \text { h } \quad-6.444778-5.77804821 .171788 \\
& \mathbf{5}^{\prime}
\end{aligned}
$$$$
\text { ZPE at B1: } 0.485258 \text { (a.u.) }
$$$$
\text { Total QM/MM energy at B2: -3236.439404 (a.u.) }
$$$$
\mathrm{Fe} \quad-3.123466-12.30413520 .160321
$$$$
\text { P } \quad-5.887605-11.54146721 .630959
$$$$
\text { C } \quad-4.833806-11.59159723 .072944
$$$$
\text { C } \quad-3.436848-12.11865323 .186454
$$$$
\text { O } \quad-2.858777-12.52384321 .987871
$$$$
\text { C } \quad-2.540394-11.08837823 .909041
$$$$
\text { O } \quad-6.976579-12.61547421 .788837
$$$$
\text { O } \quad-5.009879-11.82569520 .351830
$$$$
\text { O } \quad-6.495633-10.09612821 .571118
$$$$
\text { H } \quad-4.903541-9.14721822 .047268
$$$$
\text { H } \quad-5.281928-11.22125424 .003151
$$$$
\text { H } \quad-1.779894-10.22902220 .575829
$$$$
\text { H } \quad-2.877470-10.95517024 .950600
$$

H $\quad-3.813803-18.00404721 .917216$

H $\quad-3.646365-18.73408320 .318838$

N $\quad-4.051235-16.40329818 .912782$

H $\quad-4.285495-17.07153518 .171578$

C $\quad-4.048545-16.63843320 .284076$

C $\quad-3.710887-15.11282218 .697342$

H $\quad-3.663997-14.66757417 .707507$

N $\quad-3.480063-14.49130719 .842076$

C $\quad-3.684364-15.42586920 .837615$

H $\quad-3.539265-15.16814721 .884214$

C $\quad 1.479720-12.59005118 .647311$

H $\quad 0.859666-12.10043017 .878791$

H $\quad 1.164357-13.64321518 .696102$

C $\quad 1.287118-11.88260819 .983181$

H $\quad 1.696633-12.47380820 .819606$

H $\quad 1.796030-10.90866720 .021797$

C $\quad-0.177263-11.60041820 .279429$

O $\quad-0.428877-10.56565720 .938223$

O $\quad-1.052061-12.39893219 .802997$

O $\quad-2.706707-10.14498420 .105721$

O $\quad-4.039299-8.69488921 .999296$

H $\quad-2.477214-9.81333719 .215735$

H $\quad-3.620781-9.17815121 .255333$

C $\quad-5.866593-6.54615820 .714577$

H $\quad-5.334375-6.10550519 .861357$

H $\quad-5.129864-6.94933321 .418298$

N $\quad-6.696042-7.69465820 .212269$

H $\quad-6.541996-8.59243620 .745596$

H $\quad-7.721290-7.50365520 .236591$ 
H $\quad-6.487792-7.91626619 .217032$

h $\quad-5.441349-12.26099914 .045056$

h $\quad-5.236880-18.41732820 .906995$

h $\quad 2.480883-12.55253618 .217972$

h $\quad-6.484556-5.76738221 .161453$

TS5'

ZPE at B1: 0.485214 (a.u.)

Total QM/MM energy at B2: -3236.405956 (a.u.)

$\mathrm{Fe} \quad-3.244294-12.29097420 .123547$

P $\quad-5.893153-11.56059121 .576668$

C $\quad-4.779368-11.58735222 .990612$

C $\quad-3.459153-12.08193323 .243356$

O $\quad-3.221978-12.38131221 .945458$

C $\quad-2.497394-11.06342623 .876364$

O $\quad-6.962909-12.64184521 .807452$

O $\quad-5.148565-11.80471920 .207092$

O $\quad-6.508117-10.11451021 .588801$

H $\quad-4.920926-9.17258422 .045834$

H $\quad-5.314023-11.10439823 .836464$

H $\quad-1.904431-10.21564020 .558157$

H $\quad-2.702555-10.98241424 .956849$

H $\quad-1.459047-11.39338523 .733335$

H $\quad-2.633676-10.08063623 .403736$

H $\quad-3.460741-13.00338923 .878925$

C $\quad-5.220913-11.49495314 .744185$

H $\quad-4.920577-10.56028014 .239474$

H $\quad-6.193562-11.28507315 .215038$

N $\quad-2.901654-12.05914915 .741066$

H $\quad-2.336358-12.11557714 .891301$

C $\quad-4.271317-11.85411715 .847235$

C $\quad-2.403105-12.23412416 .985138$

H $\quad-1.355027-12.43247517 .190198$

N $\quad-3.364586-12.14929217 .894497$

C $\quad-4.530335-11.91326517 .199439$

H $\quad-5.470738-11.77672417 .728645$

C $\quad-4.271533-18.00115520 .909603$

H $\quad-3.857126-18.00478321 .929227$

H $\quad-3.682426-18.73390120 .331019$

N $\quad-4.066379-16.41566618 .920236$

H $\quad-4.278705-17.09062818 .178529$

C $\quad-4.096352-16.64107220 .292929$

C $\quad-3.743983-15.12104418 .704079$

H $\quad-3.674396-14.68152817 .712900$
$-3.555129-14.48695119 .849531$

C $\quad-3.770004-15.41763120 .846689$

H $\quad-3.666871-15.15098321 .896068$

C $\quad 1.382504-12.59163418 .695549$

H $\quad 0.758353-12.11735117 .920959$

H $\quad 1.073240-13.64566418 .760745$

C $\quad 1.183173-11.86229920 .019085$

H $\quad 1.615408-12.42310520 .865289$

H $\quad 1.671480-10.87614520 .032174$

C $\quad-0.286748-11.60900720 .325260$

O $\quad-0.554985-10.59201221 .007912$

O $\quad-1.149492-12.41028319 .837482$

O $\quad-2.800621-10.13310520 .051732$

O $\quad-4.065212-8.69982822 .027381$

H $\quad-2.533924-9.81603319 .167195$

H $\quad-3.648807-9.09458321 .234252$

C $\quad-5.891195-6.56385720 .733325$

H $\quad-5.340187-6.13610319 .885348$

$\mathrm{H} \quad-5.170627-6.96519621 .454589$

N $\quad-6.721370-7.70975120 .226518$

H $\quad-6.572432-8.61130220 .757377$

H $\quad-7.746310-7.51679620 .246313$

H $\quad-6.505594-7.93039619 .232383$

h $\quad-5.448701-12.26790314 .010204$

h $\quad-5.275818-18.42477620 .914362$

h $\quad 2.383204-12.55418518 .265126$

h $\quad-6.506932-5.77425121 .163971$

$6^{\prime}$

ZPE at B1: 0.494711 (a.u.)

Total QM/MM energy at B2: -3236.484734 (a.u.)

$\mathrm{Fe} \quad-3.467703-12.17625319 .638744$

P $\quad-5.705533-11.46333321 .814652$

C $\quad-4.162769-11.63414722 .799783$

C $\quad-4.082248-12.42987924 .033082$

O $\quad-3.514865-12.92693222 .819776$

C $\quad-3.127292-12.07734325 .142148$

O $\quad-6.714800-12.53594122 .237988$

O $\quad-5.275231-11.66913120 .310867$

O $\quad-6.171504-9.99659122 .073907$

H $\quad-4.660691-9.00762822 .275495$

H $\quad-3.470909-10.78614722 .711498$

$\mathrm{H} \quad-2.229895-10.09429320 .439535$

H $\quad-3.633403-11.44909225 .893542$ 


\begin{tabular}{|c|c|c|c|}
\hline $\mathrm{H}$ & $-2.762272-12.98271325 .654353$ & $\mathrm{H}$ & $-6.450918-8.17101319 .492850$ \\
\hline $\mathrm{H}$ & $-2.259941-11.52849224 .745204$ & $\mathrm{~h}$ & $-5.550147-12.27429013 .639925$ \\
\hline $\mathrm{H}$ & $-4.985473-12.97790324 .331386$ & $\mathrm{~h}$ & $-5.320066-18.34063120 .887936$ \\
\hline $\mathrm{C}$ & $-5.370407-11.44765014 .327257$ & $\mathrm{~h}$ & $2.110024-12.53053918 .356577$ \\
\hline $\mathrm{H}$ & $-5.110160-10.52322813 .784288$ & $\mathrm{~h}$ & $-6.531837-5.83320221 .213581$ \\
\hline $\mathrm{H}$ & $-6.352938-11.26275614 .786006$ & & \\
\hline $\mathrm{N}$ & $-3.035497-11.88821015 .353252$ & $7^{\prime}$ & \\
\hline $\mathrm{H}$ & $-2.449326-11.93040814 .517089$ & $\mathrm{ZP}$ & at B1: 0.487257 (a.u.) \\
\hline $\mathrm{C}$ & $-4.410690-11.73557515 .438728$ & Tot & QM/MM energy at B2: -3237.395741 (a.u.) \\
\hline $\mathrm{C}$ & $-2.551420-12.06352016 .603874$ & $\mathrm{Fe}$ & $-3.180528-12.37494920 .090428$ \\
\hline $\mathrm{H}$ & $-1.497784-12.22578316 .815820$ & $\mathrm{P}$ & $-5.929443-11.65251421 .636070$ \\
\hline $\mathrm{N}$ & $-3.529380-12.02677117 .496374$ & $\mathrm{C}$ & $-4.866749-11.87850323 .063258$ \\
\hline $\mathrm{C}$ & $-4.688702-11.82491216 .783340$ & $\mathrm{C}$ & $-3.386736-12.09843623 .098853$ \\
\hline $\mathrm{H}$ & $-5.656220-11.74154317 .273443$ & $\mathrm{O}$ & $-2.850792-12.56324721 .902734$ \\
\hline $\mathrm{C}$ & $-4.332325-17.88013820 .868412$ & $\mathrm{C}$ & $-2.661799-10.81318023 .562241$ \\
\hline $\mathrm{H}$ & $-3.919512-17.82397421 .887236$ & $\mathrm{O}$ & $-7.028209-12.72399321 .676767$ \\
\hline $\mathrm{H}$ & $-3.713514-18.61389820 .322463$ & $\mathrm{O}$ & $-5.040601-11.78908220 .325083$ \\
\hline $\mathrm{N}$ & $-4.183328-16.38039318 .810015$ & $\mathrm{O}$ & $-6.503979-10.21107921 .755346$ \\
\hline $\mathrm{H}$ & $-4.367092-17.09176418 .095170$ & $\mathrm{H}$ & $-5.347085-11.69224724 .032008$ \\
\hline $\mathrm{C}$ & $-4.210632-16.54294220 .190019$ & $\mathrm{H}$ & $-2.939260-10.59156224 .604849$ \\
\hline $\mathrm{C}$ & $-3.930775-15.08062718 .537435$ & $\mathrm{H}$ & $-1.573841-10.95614823 .489584$ \\
\hline $\mathrm{H}$ & $-3.878083-14.68316317 .526173$ & $\mathrm{H}$ & $-2.954548-9.95792622 .934123$ \\
\hline $\mathrm{N}$ & $-3.777509-14.38542519 .649678$ & $\mathrm{H}$ & $-3.184648-12.86253223 .885214$ \\
\hline $\mathrm{C}$ & $-3.947983-15.28051120 .689460$ & $\mathrm{C}$ & $-5.274515-11.29188614 .733760$ \\
\hline $\mathrm{H}$ & $-3.863342-14.94531221 .721831$ & $\mathrm{H}$ & $-4.913385-10.39290014 .205405$ \\
\hline $\mathrm{C}$ & $1.107577-12.56491918 .783172$ & $\mathrm{H}$ & $-6.234699-11.01181315 .191973$ \\
\hline $\mathrm{H}$ & $0.484689-12.10551217 .998713$ & $\mathrm{~N}$ & $-2.964247-11.71230815 .733788$ \\
\hline $\mathrm{H}$ & $0.800881-13.61870818 .859961$ & $\mathrm{H}$ & $-2.427090-11.57637614 .877494$ \\
\hline $\mathrm{C}$ & $0.886209-11.82171920 .098769$ & $\mathrm{C}$ & $-4.343517-11.67740715 .840989$ \\
\hline $\mathrm{H}$ & $1.322361-12.36637120 .953560$ & $\mathrm{C}$ & $-2.447829-11.99091616 .950291$ \\
\hline $\mathrm{H}$ & $1.363263-10.82876720 .104188$ & $\mathrm{H}$ & $-1.385186-12.09311817 .153178$ \\
\hline $\mathrm{C}$ & $-0.594980-11.58699320 .396903$ & $\mathrm{~N}$ & $-3.414301-12.13083117 .848919$ \\
\hline $\mathrm{O}$ & $-0.896846-10.59432721 .097773$ & $\mathrm{C}$ & $-4.600789-11.94343717 .168150$ \\
\hline $\mathrm{O}$ & $-1.437074-12.38986119 .876817$ & $\mathrm{H}$ & $-5.559233-11.98913417 .681562$ \\
\hline $\mathrm{O}$ & $-3.017799-9.93139619 .830524$ & $\mathrm{C}$ & $-4.143253-18.04754620 .853159$ \\
\hline $\mathrm{O}$ & $-3.794270-8.55529622 .199079$ & $\mathrm{H}$ & $-3.698235-18.05832021 .859527$ \\
\hline $\mathrm{H}$ & $-2.595456-9.56702419 .026810$ & $\mathrm{H}$ & $-3.578005-18.78213120 .254370$ \\
\hline $\mathrm{H}$ & $-3.537589-8.80441021 .291204$ & $\mathrm{~N}$ & $-3.981743-16.45290218 .868061$ \\
\hline $\mathrm{C}$ & $-5.910863-6.64366220 .831989$ & $\mathrm{H}$ & $-4.148861-17.13679518 .120390$ \\
\hline $\mathrm{H}$ & $-5.394958-6.27482019 .935085$ & $\mathrm{C}$ & $-3.983133-16.68686020 .239606$ \\
\hline $\mathrm{H}$ & $-5.152628-6.94439621 .564213$ & $\mathrm{C}$ & $-3.684891-15.15212818 .652384$ \\
\hline $\mathrm{N}$ & $-6.699413-7.86729320 .457282$ & $\mathrm{H}$ & $-3.630184-14.70453017 .664353$ \\
\hline $\mathrm{H}$ & $-6.509879-8.70970421 .084158$ & $\mathrm{~N}$ & $-3.502119-14.52064919 .802633$ \\
\hline $\mathrm{H}$ & $-7.729387-7.70625020 .438429$ & $\mathrm{C}$ & $-3.681295-15.46186220 .797832$ \\
\hline
\end{tabular}


H $\quad 0.819392-12.27932817 .888040$

H $\quad 1.117354-13.79256118 .744905$

C $\quad 1.226666-11.99298219 .989534$

H $\quad 1.611592-12.56889520 .848339$

H $\quad 1.755469-11.02643020 .015249$

C $\quad-0.239364-11.67868520 .272054$

O $\quad-0.484783-10.69719421 .013340$

O $\quad-1.119017-12.40616219 .704452$

O $\quad-2.737019-10.15069020 .037361$

H $\quad-2.335992-9.91077519 .178395$

H $\quad-1.894979-10.26538820 .609908$

O $\quad-4.606558-8.44628721 .328593$

H $\quad-5.265292-9.14846421 .590434$

H $\quad-3.966081-8.93702120 .776262$

C $\quad-5.858866-5.79407621 .334889$

H $\quad-5.054782-5.45674820 .668160$

H $\quad-5.404038-6.27567322 .205774$

N $\quad-6.638575-6.82483720 .602368$

H $\quad-6.911475-6.47443319 .666133$

$\mathrm{H} \quad-6.011833-7.66898620 .545396$

H $\quad-7.544129-7.04541721 .073674$

h $\quad-5.524324-12.07549714 .018487$

h $\quad-5.152451-18.45785920 .888230$

h $\quad 2.440057-12.71703718 .257698$

h $\quad-6.468353-4.94012521 .630457$

\section{TS6'}

ZPE at B1: 0.485917 (a.u.)

Total QM/MM energy at B2: -3237.374914 (a.u.)

$\mathrm{Fe} \quad-3.163756-12.41409319 .998043$

P $\quad-5.869942-11.78421021 .656492$

C $\quad-4.713972-12.41696022 .923850$

C $\quad-3.314277-11.96453623 .150076$

O $\quad-2.948482-12.62206121 .952058$

C $\quad-2.994900-10.48484323 .253662$

O $\quad-7.056191-12.75505021 .699128$

O $\quad-5.079826-11.84090420 .295951$

O $\quad-6.264691-10.32625222 .006329$

H $\quad-4.959595-13.41144123 .313134$

H $\quad-3.337199-10.12119324 .236499$

$\mathrm{H} \quad-1.908083-10.33603223 .171458$

H $\quad-3.490017-9.88044322 .484706$
$-2.858121-12.48708724 .015277$

C $\quad-5.284630-11.34875114 .676923$

H $\quad-4.949064-10.43505314 .157715$

H $\quad-6.244729-11.09840815 .152264$

N $\quad-2.952029-11.68422015 .658516$

$\mathrm{H} \quad-2.428293-11.48445714 .805464$

C $\quad-4.330638-11.73263815 .765662$

C $\quad-2.420625-11.97155616 .868065$

$\mathrm{H} \quad-1.352798-12.01051717 .068031$

N $\quad-3.375864-12.20041517 .759257$

C $\quad-4.570014-12.06323517 .081442$

H $\quad-5.526572-12.17976917 .587166$

C $\quad-4.144880-18.11111820 .838690$

H $\quad-3.700750-18.12409221 .845547$

H $\quad-3.589267-18.85603920 .243898$

N $\quad-3.952633-16.52543318 .848206$

$\mathrm{H} \quad-4.120687-17.20868718 .100552$

C $\quad-3.966863-16.75525720 .219759$

C $\quad-3.645254-15.22613818 .632809$

$\mathrm{H} \quad-3.577484-14.78084017 .643798$

N $\quad-3.468895-14.59228919 .782006$

C $\quad-3.660603-15.53041120 .776064$

H $\quad-3.552503-15.26534121 .824284$

C $\quad 1.465893-12.75384418 .716524$

H $\quad 0.827339-12.34504517 .917982$

H $\quad 1.171090-13.80476418 .841711$

C $\quad 1.253456-11.94465519 .993695$

H $1.693620-12.44610120 .872541$

H $\quad 1.727865-10.95029219 .950063$

C $\quad-0.225988-11.70068720 .291105$

O $\quad-0.516507-10.72102621 .028000$

O $\quad-1.067329-12.46909819 .737686$

O $\quad-2.742062-10.17933719 .934560$

H $\quad-2.308949-9.96118619 .085312$

H $\quad-1.925755-10.27852920 .538580$

O $\quad-4.723829-8.45910221 .056672$

H $\quad-5.263316-9.20444521 .453482$

H $\quad-4.073699-8.92968820 .498017$

C $\quad-5.911664-5.79239121 .351767$

H $\quad-5.100398-5.46236420 .689868$

H $\quad-5.468219-6.29306522 .218167$

N $\quad-6.714762-6.78966820 .601220$

$\mathrm{H} \quad-7.010186-6.39475019 .687837$

H $\quad-6.079710-7.63111320 .488155$ 
H $\quad-7.605626-7.03143821 .088481$

h $\quad-5.529571-12.12809613 .955333$

h $\quad-5.159730-18.50725220 .873672$

h $\quad 2.465259-12.72559018 .282318$

h $\quad-6.505494-4.93030121 .655466$

\section{$8^{\prime}$}

ZPE at B1: 0.496241 (a.u.)

Total QM/MM energy at B2: -3237.447780 (a.u.)

$\mathrm{Fe} \quad-3.270265-12.45571119 .788151$

$\mathrm{P} \quad-5.826798-11.92231821 .711250$

C $\quad-4.511612-12.65600622 .795444$

C $\quad-3.336290-11.97277723 .371716$

O $\quad-3.202429-12.78266722 .191062$

C $\quad-3.050505-10.50012023 .296258$

O $\quad-7.031094-12.85390221 .836144$

O $\quad-5.175963-11.95141420 .273425$

O $\quad-6.125360-10.47094722 .168519$

H $\quad-4.848572-13.57729323 .294091$

H $\quad-3.366914-10.03506224 .244309$

H $\quad-1.971768-10.33740923 .155718$

H $\quad-3.591628-9.99904822 .486740$

H $\quad-2.889838-12.46870324 .247474$

C $\quad-5.361639-11.37312714 .486656$

H $\quad-5.047885-10.45859513 .956089$

H $\quad-6.334107-11.14687914 .948111$

N $\quad-3.031859-11.59555015 .498800$

$\mathrm{H} \quad-2.512223-11.33590314 .657587$

C $\quad-4.404692-11.71711315 .584572$

C $\quad-2.503244-11.89698916 .707595$

H $\quad-1.436443-11.87907216 .918915$

$\mathrm{N} \quad-3.455641-12.20736217 .575927$

C $\quad-4.644753-12.10464316 .884262$

H $\quad-5.604854-12.30551817 .354274$

C $\quad-4.205823-18.17371520 .852985$

H $\quad-3.768052-18.18301621 .862627$

H $\quad-3.654969-18.92987820 .267977$

$\mathrm{N} \quad-4.005097-16.60780618 .851213$

H $\quad-4.165786-17.29619218 .106613$

C $\quad-4.017691-16.82512020 .221618$

C $\quad-3.714837-15.30485318 .627004$

H $\quad-3.639541-14.86753917 .633613$

N $\quad-3.546223-14.65734519 .769355$

C $\quad-3.723364-15.59189220 .767330$
$-3.617139-15.32742621 .816158$

C $\quad 1.331354-12.75943718 .762594$

H $\quad 0.688998-12.35384117 .964906$

H $\quad 1.044792-13.81273618 .885075$

C $\quad 1.111152-11.95612720 .044087$

H $\quad 1.553923-12.46088920 .919854$

H $\quad 1.580176-10.95903720 .006018$

C $\quad-0.371330-11.72195920 .346223$

O $\quad-0.676242-10.72260021 .045502$

O $\quad-1.204102-12.53367219 .837410$

O $\quad-2.865893-10.14910719 .802107$

$\mathrm{H} \quad-2.382005-9.90886618 .986955$

H $\quad-2.105237-10.25387820 .454741$

O $\quad-4.884529-8.55517320 .916163$

H $\quad-5.323074-9.30930621 .408091$

H $\quad-4.199083-9.00186920 .376934$

C $\quad-5.990789-5.83024221 .369564$

H $\quad-5.181464-5.51931120 .695605$

H $\quad-5.548018-6.33735822 .232959$

N $\quad-6.827706-6.80954020 .631466$

H $\quad-7.137379-6.39506119 .730688$

H $\quad-6.207324-7.65998820 .485472$

H $\quad-7.704228-7.05539221 .142066$

h $\quad-5.576958-12.17549313 .781027$

h $\quad-5.226461-18.55517620 .882167$

h $\quad 2.330333-12.72289618 .328114$

h $\quad-6.563046-4.95424621 .674884$ 